\title{
Ancora intorno a completamenti di spazi uniformi.
}

\author{
Memoria di Grovanni Aquaro (a Bari)
}

Sunto. - $\grave{E}$ contenuto nell' introduzione.

Introduzione. - Nel presente lavoro si prosegue lo studio degli spazii uniformizzabili $\mathscr{A}_{a}$-completi, iniziato in una Nota, apparsa in questi Annali [4], alla quale si rinvia per la terminologia ed il simbolismo qui usati.

Al concetto di spazio $\mathscr{A}_{a}$-completo fa riscontro il concetto di $\mathscr{A}_{a}$-completamento ( $\$ 1$, def. 1) e nel seguente $\& 1$ viene approfondita l'analisi delle proprietà di entrambi ottenendosi, in una situazione più generale, proprietà analoghe alle piu importanti dei $Q$-spazii secondo HewITT.

Successivamente, nel $\S 2$, utilizzando alcuni risultati ottenuti dallo scrivente in [2], viene effettuata una esposizione sistematica ed autonoma dei filtri $\mathscr{\ell}$-inviluppati, $\ell$ essendo una struttura uniforme, riunendo, in un assetto unico, risultati di [11], [1], [10].

Nel $\S 3$, tramite i mezzi forniti dal $\S 2$, si stabiliscono alcune proprietà dei Q-spazii nel quadro delle tecniche sviluppate in [3], [4] e nel presente lavoro. Successivamente, si ntilizzano le conclusioni, alle quali così si perviene, per caratterizzare gli spazii uniformizzabili separati il cui $\mathscr{A}_{\omega}$-completamento, con $\omega=$ card $(\boldsymbol{N})$, è uno spazio di LINDELöF. Tali spazii sono caratterizzati dalla seguente proprietà.

$(* *)$ - ogni filtro completamente regolare verificante la proprietà della intersezione numerabile è meno fine di un filtro massimale della stessa specie.

La (**) va posta in relazione con una consimile proprietà introdotta da R. W. Bagleix e J. D. MoKnight JR. in [6]. La $(* *)$, tuttavia, sembra prestarsi meglio agli scopi prefissici consentendo di ottenere non solo la caratterizzazione di cui sopra ma anche qualche miglioramento dei risultati dei suddetti autori.

(*) Lavoro eseguito nel Gruppo di Ricerca n. 9 del Comitato Nazionale per la Matematica del Consiglio Nazionale delle Ricerehe, per l'anno 1961-62. 


\section{$\S 1$ - Aa-Completamenti ed ulteriori proprietà degli spazii $\mathscr{A l}_{a}$-completi}

1. Si è osservato in [4] $\S 2$, prop. 7 , che, se $E$ è uno spazio uniformiz* zabile separato (= di HaUSDORFF), se a è numero cardinale infinito e se $\mathscr{A} \mathfrak{a}(E)$ è la a-struttura uniforme su $E([3] \S 3$, def. 4), esiste uno spazio uniformizzabile separato $\mathcal{A}_{a}$-completo ([4] $\left.\S 2\right)$, che denotiamo con

$$
v_{\mathfrak{a}}(E)
$$

(che in [4] si denotava, invece, con $v_{\mathfrak{a}}(E)$ ), tale che $E$ sia (ingettivamente) immerso ed ovunque denso in $v_{\mathfrak{a}}(E) \theta$, denotata con $\mathcal{L}_{\mathfrak{a}}\left(v_{\mathfrak{a}}(E)\right)$ la a-struttura uniforme di $v_{\mathfrak{a}}(E)$ e con $\mathscr{A}_{\mathfrak{a}}\left(U_{\mathfrak{a}}(E)\right)_{E}$ la struttura uniforme indotta da $\mathscr{A}_{\mathfrak{a}}\left(v_{\mathfrak{a}}(E)\right)$ su $E$, risulti

$$
\mathscr{A}_{\mathfrak{a}}(E)=\mathscr{A}_{\mathfrak{a}}\left(\cup_{\mathfrak{a}}(E)\right)_{E}
$$

DEF. 1 - Lo spazio uniformizzabile separato va $(E)$ ora descritto chiamasi Aa-completamento di $E$.

Si deve subito segnalare la seguente proprietà caratterizzante $v_{\mathfrak{a}}(E)$.

Prop. 1 - Se E è uno spazio uniformizzabile separato, se a è un numero cardinale infinito e se $v_{\mathfrak{a}}(E)$ è l's $l_{\mathfrak{a}}$-completamento di $E$ (def. 1), val $(E)$ è uno ed è omeomorfo a ciascuno degli spazii uniformizzabili separali $E^{*}$ che sono Aa-completi ([4]\$2, def. 2) ed a ciascuno dei quali si può associare un'ingezione $j^{*}$ di $E$ in $E^{*}$ con le seguenti condizioni:

a) $j^{*}(E)$ è ovunque denso in $E^{*}$,

b) detta $k^{*}$ la bigezione (ridolta) di $E$ su $j^{*}(E)$ definita da $j^{*}$, tale $k^{*}$ è un omeomorfismo di $E$ su $j^{*}(E)$.

c) per ogni applicazione continua $f$ di $E$ in uno spazio uniformizzabile separato $\mathscr{L}_{a-c o m p l e t o} X$, esiste una (ed una sola) applicazione continua $\bar{f} d i$ $E^{*}$ in $X$, tale che $\bar{f} \circ j^{*}=f$.

Osservaztone - Si può, equivalentemente, descrivere $E^{*}$ come uno spazio uniformizzabile separato in cui $E$ sia contenuto e ovunque denso e tale che ogni applicazione continua $f$ di $E$ in uno spazio uniformizzabile separato Aa-completo $X$, possa prolungarsi in una applicazione continua $\bar{f}$ di $E^{*}$ in $X$. In appresso di frequente le proprietà di $E^{*}$ descritte da a), b) e c) della prop. 1 , si intederanno espresse in questi termini.

Prop. 2 - Se E, a e val $E)$ sono quelli della prop. 1, le seguenti proposizioni sono equivalenti: 
a) $E$ è sla-completo (cfr. [4] §2, def. 2)

b) risulta $E=v_{\mathfrak{a}}(E)$

Dru. a) implica b). In forza di (1), $\left(E, \mathscr{A}_{\mathfrak{a}(E))}(E)\right.$ uno sottospazio uniforme completo dello spazio uniforme $\left(v_{\mathfrak{a}}(E), \mathscr{A}_{\mathfrak{a}}\left(v_{\mathfrak{a}}(E)\right)\right)$ : ciò, poichè $v_{\mathfrak{a}}(E)$ è separato ( $=$ di HadSDORFF), implica che $E \dot{\theta}$ chiuso in $v_{\mathfrak{a}}(E)$ e quindi, $E$ essendo ovunque denso in $v_{\mathfrak{a}}(E)$, si ha la b).

b) implica a). 亡̀ orvio, dato che $v_{\mathfrak{a}}(E)$ ò $\mathscr{A}_{\mathfrak{a}}$-completo.

In tema di spazii $\mathscr{A}_{\mathfrak{a}}$-completi, torna a proposito segnalare alcuni esempii che dovremo, almeno in parte, utilizzare. All' nopo premettiamo un lemma.

Lemma 1. - Supponiamo che E sia uno spazio topologico e che, supposto a un numero cardinale infinito, $E$ verifichi la proprietà:

$\left(L_{\mathfrak{a}}\right)-$ se $\left(U_{i}\right)_{i \in I}$ è un qualunque ricovrimento aperto di $E$ esiste una parte $H$ dell' insieme degli indici $I$, tale che card. $(H) \leq \mathfrak{a}$ e tale che la sottofamiglia $\left(U_{i}\right)_{\in \in H}$ sia un ricovrimento di $E$.

Allora, se $\left(A_{\lambda}\right)_{\lambda \in L} \dot{e}$ una qualunque famiglia localmente finita di parti non vuote di $E$ risulta card $(L) \leq \mathfrak{a}$.

DrM. - Esiste un ricovrimento aperto $\left(U_{t}\right)_{\iota \in I}$ di $E$ tale che, per ogni $: \in I$, l'insieme $L_{\imath}^{*}$ dei $\lambda \in L$ tali che $A_{\lambda} \cap U_{t} \neq \varnothing$ sia finito. In forza di $\left(L_{\mathfrak{a}}\right)$ esiste una parte $H$ di $I$ tale che card $(H) \leq \mathfrak{a}$ e la sottofamiglia $\left(U_{t}\right)_{\in}$ ricopra $E$.

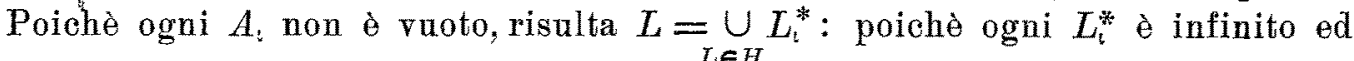
è card $(H) \leq \mathfrak{a}$, si ha card $(L) \leq \mathfrak{a}$.

Osservazione 1. - Se è $\mathfrak{a}=$ card $(\boldsymbol{N}), E$ verifica $\left(L_{\mathfrak{a}}\right)$ se e solo se esso è uno spazio di LINDELöF.

Osservazione 2. - Se $E$ è uniformizzabile e verifica la $\left(L_{\mathfrak{a}}\right)$, la a-struttura uniforme di $E$ è identica alla struttura uniforme universale ([3] $\S 3$, def. 4). Pertanto, se esiste una struttura uniforme di spazio completo su $E$, compatibile con la topologia di $E$, poichè allora la struttura uniforme uni. versale è una struttura di spazio completo, in forza della def. 2 \& 2 di [4], $E$ risulta $\mathscr{A}_{a}$-completo.

Ossentazione 3. - Se $E$ è uno spazio regolare paracompatto e verifica la $\left(L_{\mathfrak{a}}\right)$ esso, per la osservazione 2, è $\mathscr{A}$ a-completo, poichè, come è noto, la sua struttura uniforme universale è una struttura di spazio completo.

Osservazione 4. - Supponiamo che $E$ sia uno spazio di LindelöF (cfr. osservazione 1) regolare. Come è noto, $E$ è paracompatto ed allora, in forza della osservazione $3, E$ è $\mathscr{A}_{\omega}$-completo, con $\omega=$ card $(N)$.

Osservazione 5. - Se lo spazio topologico $E$ (nessuna restrizione) ha una base per la sua topologia $\left(T_{\lambda}\right)_{\lambda \in L}$ tale che card $(L) \leqslant \mathfrak{a}$, allora $E$ verifica la proprietà $\left(L_{\mathfrak{a}}\right)$ del lemma 1. 
Ossevazione 6. - Se $E$ è uno spazio quasi-metrizzabile ed ha una base del tipo descritto nell'osservazione 5, allora $E$ verifica la $\left(L_{\mathfrak{a}}\right)$ e quindi, essendo regolare paracompatto, risulta $\mathcal{A l}_{\mathbf{a}}$-completo.

Osservazione 7. - Ogni spazio quasi-metrizzabile dotato di base numerabile (o, che è lo stesso, regolare e dotato di base numerabile) $E$ risulta $\mathscr{A}_{\mathbf{a}}$-completo.

Nelle precedenti osservazioni sono contenuti i preannunciati esempii di spazii $\mathscr{S t a}_{\mathrm{a}}$-ompleti.

Osservazione 8. - Se lo spazio uniformizzabile $E$ è $A$ a-completo ogni spazio topologico omeomorfo ad esso gode delle stesse proprietà. Di questa ovvia osservazione ci serviremo senza esplicito riferimento.

2. - Per stabilire la prop. 3 qui appresso conviene isolare il seguente lemma :

LAMMa 2. - Supponiamo che E sia uno spazio uniformizzabile, che a sia un numero cardinate infinito e che $\mathscr{A}(E)$ sia la a-struttura uniforme su $E$. Allora esiste una famiglia $\left(\eta_{\beta}\right)_{\beta \in B}$ di strulture uniformi quasi-metrizzabili su $E$, tutte meno fini di $\mathscr{A}_{\mathfrak{a}}(E)$ tali che per ogni $\beta \in B$, la topologia (di spazio quasi-metrizzabile) $\tau_{\beta}$ dedotta da $\eta_{\beta}$ abbia una base $\left(T_{\beta \lambda}\right\rangle_{\lambda \in L_{\beta}}$ tale che card $\left(L_{\beta}\right) \leq \mathfrak{a}$ e tale che $\mathcal{L}_{\mathfrak{a}}(E)$ sia la struttura uniforme estrema superiore della famiglia $\left(M_{\beta}\right)_{\beta \in B}\left(^{1}\right)$ cioè $\mathscr{A}_{\mathbf{a}}(E)=\sup _{\beta \in B} \prod_{\beta}$.

DIM. - La tesi viene stabilita quasi immediatamente utilizzando il lemma 2 n. 2 di [5].

Supponiamo che $\left(V_{\beta}\right)_{\beta \in B}$ sia una rappresentazione parametrica dell'insieme delle adiacenze di $E$ per $\mathscr{L}_{\mathfrak{a}}(E):$ se è $\beta \in B$, per definizione di $\mathscr{L}_{\mathfrak{a}}(E)$ esiste un ricovrimento aperto, localmente finito ed $\mathscr{\ell}$-riducibile $\left(U_{\imath}\right)_{t \in I}$ di $E$ tale che

$$
\begin{gathered}
\text { card }(I) \leq \mathfrak{a} \\
\bigcup_{\iota \in I}\left(U_{\mathfrak{l}} \times U_{!}\right) \subset V_{\beta} .
\end{gathered}
$$

Per il lemma testé citato di [5] esiste una struttura uniforme quasimetrizzabile $M_{\beta}$ su $E$, meno fine di $s(t a)$, tale che la topologia $\tau_{\beta}$ su $E$ dedotta da $M_{\beta}$ abbia una base $\left(T_{\beta \lambda}\right)_{\lambda \in L_{\beta}}$ tale che card $\left(L_{\beta}\right) \leq a$ e tale che esista un'adiacenza $W$ di $E$ per $\nearrow_{\beta}$ tale che $(W(x))_{x \in E}$ sia un raffinamento

(1) Ciò̀ la meno fine delle strutture uniformi su $E$ più fini di ciascuna delle $\mathscr{M}_{\beta}$ al variare di $\beta$ in $B$. 
di $\left(U_{\imath}\right)_{i \in I}$. Supponiamo che $W$ sia simmetrica. Allora si ha:

$$
W \subset W \circ W=\bigcup_{x \in E}(W(x) \times W(x)) \subset \bigcup_{t \in I}\left(U_{t} \times U_{\imath}\right) \subset V_{\beta} .
$$

Dunque $V_{\beta}$ è un'adiacenza di $E$ per $\dddot{M}_{\beta}$ e quindi $\mathscr{A}_{a}(E)$ è meno fine di $\sup _{\beta \in B} \prod_{\beta}$. D'altra parte ogni $\prod_{\beta}$ è meno tine di $\mathscr{A}_{a}(E)$ e quindi, ovviamente, la tesi è dimostratata.

La proposizione qui appresso è fondamentale per gli obbiettivi che sono in vista in quanto segue.

Prop. 3. - Nelle ipotesi per $E$, a e $\mathcal{A l}_{\mathfrak{a}}(E)$ fissate nel lemma 2, esiste una famiglia $\left(E_{\beta}\right)_{\beta \in B}$ di spazii uniformi quasi-metrizzabili ciascuno dei quali ha una base per la sua topologia $\tau_{\beta}$ che denotiamo con $\left(T_{\lambda \beta}\right)_{\lambda \in L_{\beta}}$, tale che card $\left(L_{\beta}\right) \leq \mathfrak{a}$ e tale che lo spazio uniforme $\left(E, \mathscr{A}_{\mathfrak{a}}(E)\right)$ sia isomorfo (nel senso della teoria degli spazii uniformi) con un sottospazio uniforme dello spazio uniforme prodotto

$$
\prod_{\beta \in B} E_{\beta}
$$

Inoltre se E è separato (= di Hausdorff) ogni $E_{\beta}$ pù̀ essere supposto, in particolare, uno spazio metrizzabile.

DrM. - L'asserto viene stabilito, tramite il lemma 2, con una argomentazione che, salvo una precisazione, riproduce quella adoperata in [10] prop. 34.6 alla quale si rinvia il lettore per i particolari di dettaglio.

Come il lemma 2 consente, si assuma una famiglia $\left(\mathbb{M}_{\beta}\right)_{\beta \in B}$ di strutture uniformi quasi-metrizzabili su $E$, tutte meno fini di $\mathscr{A}_{a}(E)$ tali che, per ogni $\beta \in B$, la topologia (di spazio quasi-metrizzabile) $\tau_{\beta}$ su $E$ dedotta di $\mathscr{M}_{\beta}$ abbia una base $\left(T_{\beta \lambda}\right)_{\lambda \in L_{\beta}}$ tale che card $\left(L_{\beta}\right) \leq \mathfrak{a}$ e tale che $\mathcal{A}_{\mathfrak{a}}(E)$ risulti la struttura uniforme estrema superiore della famiglia $\left(M_{\beta}\right)_{\beta \in B}$.

Per ogni $\beta \in B$ sia $E_{\beta}=E$ e muniamo $E_{\beta}$ della struttura uniforme $m_{\beta}$ (cioè consideriamo lo spazio uniforme $\left(E_{\beta}, M_{\beta}\right)$ ). Consideriamo lo spazio uniforme prodotto

$$
P=\underset{\beta \in B}{\pi} E_{\beta}
$$

(munito della struttura aniforme prodotto delle $\mathscr{M}_{\beta}$ ).

Per ogni $\beta \in B$ sia $\rho_{\beta}$ l'applicazione identica di $E_{\beta}$ e consideriamo la applicazione prodotto $\varphi=\underset{\beta \in B}{\times} \varphi_{\beta}$. Orviamente $\varphi$ applica bigettivamente $E$ sulla diagonale $\Delta$ di $P$ (insieme degli $\left(x_{\beta}\right)_{\beta \in B} \in P$ tali che, per un certo $x \in E$, risulti $x_{\beta}=x$ per ogni $\beta \in B$ ). Si riconosce che $\varphi$ è isomorfismo dello spazio uniforme $\left(E, \mathscr{A}_{\mathfrak{a}}(E)\right)$ su $\Delta$ munito della struttura uniforme indotta su esso dalla struttura prodotto delle $\bigcap_{\beta}$. Da ciò la prima parte della tesi. 
Per quanto concerne la seconda parte, supponiamo $E$ separato e, per ogni $\beta \in B$, consideriamo lo spazio uniforme separato associato ad $\left(E_{\beta}, M_{\beta}\right)$ e denotiamolo con $\left(E_{\beta}^{*}\right.$, $\left.\eta_{\beta}^{*}\right)$ dove, appunto, $\prod_{\beta}^{*}$ è la struttura uniforme associata alla $\prod_{\beta}$. Poichè $\mathscr{C}_{\beta}$ è una struttura uniforme quasi-metrizzabile, la $\prod_{\beta}^{*}$ è metrizzabile ed $E_{\beta}^{*}$ munito della topologia $\tau_{\beta}^{*}$ dedotta da $\prod_{\beta}^{*} \grave{e}$ uno spazio metrico.

Sia $\chi_{\beta}$ la surgezione canonica di $E_{\beta}$ sopra $E_{\beta}^{*}$ : essa è una applicazione continua, chiusa, aperta e propria $\left({ }^{2}\right)$ e poichè $E_{\beta}$ ha una base $\left(T_{\lambda}\right)_{\lambda \in L}$ tale che card $(L) \leq \mathfrak{a}$, in forza del lemma 2 , n. 2 di [5], altrettanto accade per $E_{\beta}^{*} \operatorname{con} \tau_{\beta}$.

Sia $\chi=\underset{\beta \in B}{\times} \chi_{\beta}$. Posto $\psi=\chi \circ \varphi$, come nella prop. 34.6 di [10], si riconosce che, $E$ essendo separato, $\psi$ è un isomorfismo dello spazio uniforme separato $\left(E, \mathscr{A}_{a}(E)\right)$ in un sottospazio uniforme del prodotto

$$
P^{*}=\prod_{B \in B} E^{*}
$$

munito della struttura uniforme prodotto delle $\varlimsup_{\beta}^{*}$. Da ciò la tesi.

3. - Per proseguire più agevolmente l'analisi intrapresa, converrà effettuare qualche richiamo e qualche precisazione di Algebra Topologica.

Per tutti i riferimenti d'Algebra si potrà tener presente [7].

In particolare, se $A$ è un'anello commutativo dotato di unità, dovremo adoperare il concetto di «algebra sopra $A$ » (non necessariamente dotata di unità per la moltiplicazione) conformemente alla def. $1, \S 7$, cap. II cap. cit. Per ciò che ci riguarda, $A$ sarà il corpo reale $\boldsymbol{R}$.

In primo luogo, supponiamo ohe $E$ sia un'algebra su $A$ e, detta $X$ una sua parte non vuota sia $E(X)$ la sottoalgebra generata da $X$.

Notoriamente $E(X))$ è caratterizzata dalle seguenti proprietà :

1) $-E(X)$ è una sottoalgebra di $E$ contenente $X$.

2) - se $H$ è una qualunque sottoalgebra di $E$ contenente $X$, risulta $E(X) \subset X$.

Supponiamo che $M(X)$ sia la parte di $E$ stabile per la moltiplicazione di $E$ (come anello) generata da $X$ e sia $V(M(X))$ il sottomodulo di $E$, considerato come $A$-modulo unitario in modo canonico, generato da $M(X)$. Come è ben noto si ha

$$
E(X)=\nabla(M(X))
$$

(2) Ofr. N. Bourbakr; Topologie Générale; Actual. Scient. et Ind. 1142, Hermann (Parigi), terza edizione (1961): cap. II $\$ 3$, n. 9 prop. 17 Remarque. 
Supponiamo ora, molto più particolarmente che $E$ sia un'algebra di BANACH, ciò̀ un'algebra normata, con noma \|\| , completa. Ora ̀̀ $A=R$. ([8] cap. $9, \S 3$, n. 7, def. 9).

Sia $B$ una qualunque parte di $X$ densâ in $X$, considerato come sottospazio metrico di $E$, e quindi

$$
X \subset \bar{B}
$$

e sia $E(B)$ la sottoalgebra generata da $B$. Poniamo:

$$
H=\overline{E(B)}, \quad K=\overline{E(X)} .
$$

Notoriamente $H$ e $K$ sono sottoalgebre chiuse di $E$ e, più precisamente, le più piccole (per inclusione) delle sottoalgebre chiuse di $E$ contenenti $B$ e, rispett., $X$. Risultando $B \subset E(B) \subset E(X)$ si ha $X \subset \bar{B} \subset H \subset K$ e quindi $X \subset H$, donde, $H$ essendo un'algebra, per 2) risulta $E(X) \subset H$ nonchè $K=\overline{E(X)} \subset H=H$. In conclusione è

$$
H=K
$$

Detta $M(B)$ la parte di $E$ stabile per la moltiplicazione generata da $B$ (analoga di $M(X))$ e $V(M(B))$ il sottospazio vettoriale di $E$ generato da $M(B)$ (analogo di $M(X)$ ), anche ora si ha

$$
E(B)=V(M(B))
$$

e quindi è

$$
K=\overline{V(M(B))}
$$

Denotato con $\mathbf{Q}$ l'insieme dei numeri razionali, sia

$$
M_{Q}(B)
$$

l'insieme delle combinazioni lineari, a coefficienti in $Q$, di elementi di $M(B)$. Ovviamente risulta $M_{Q}(B) \subset V(M(B))=V(M(X))$ e da ciò

$$
M_{Q}(B) \subset K
$$

Riconosciamo ora che risulta

$$
\overline{M_{Q}}(B)=K
$$

A tal fine, ricordato che $V(M(B))$ ò il sottospazio vettoriale di $E$ generato da $M(B)$, se è $x \in K$ ed $\varepsilon$ è un elemento positivo di $\boldsymbol{R}$, esistono una fami- 
glia finita $\left(m_{t}\right)_{i \in I}$ di elementi di $M(B)$ ed una famiglia finita $\left(a_{t}\right)_{t \in I}$ di elementi di $R$, tali che

$$
\left\|x-\sum_{i \in I} a_{\imath} m_{\iota}\right\|<\frac{\varepsilon}{2}
$$

Supponiamo come è, ovviamente, lecito che sia $m_{\imath} \neq 0$ per ogni $\iota \in I$ e quindi che per ogni $\imath \in I$ sia

$$
\left\|m_{\imath}\right\| \neq 0
$$

Allora, poichè $\boldsymbol{Q}$ è ovanque denso in $\boldsymbol{R}$, detto $n$ il numero degli elementi dell'insieme finito $I$, esiste un $q_{\text {t }} \in \boldsymbol{Q}$, per ogni $\downarrow \in I$, tale che

Consegue :

$$
\left|q_{t}-a_{t}\right|<\frac{\varepsilon}{2 n\left\|m_{t}\right\|}
$$

$$
\left\|x-\sum_{: \in I} q_{i} m_{\imath}\right\|<\varepsilon .
$$

e quindi $x \in \overline{M_{Q}(B)}$ e da ciò la (1).

Ora osserviamo che, come è noto, $M(B)$ è l'insieme dei composti moltiplicativamente delle famiglie finite $\left(x_{k}\right)_{0 \leq k \leq n}$ di elementi di $B$. Consegue:

$$
\operatorname{card}(M(B))=\operatorname{card}(B) .
$$

Poichè $Q$ è numerabile consegue card $\left(M_{\mathbf{Q}}(B)\right)=\operatorname{card}(M(B))$ e da ciò

$$
\operatorname{card}\left(M_{Q}(B)\right)=\operatorname{card}(B) .
$$

4. - Supponiamo che $(E, d)$ sia uno spazio metrico con distanza $d$. Sostituendo eventualmente $d$ con $d /(1+d)$, possiamo supporre $d$ limitata senza alterare la struttura uniforme di $(E, d)$.

Denotiamo con $¥(E)$ l'algebra di BANAOH delle funzioni reali continue e limitate su $(E, d)$ con la norma \|\| definita ponendo

$$
\|f\|=\sup _{x \in E}|f(x)|
$$

per ogni $f \in \mathcal{B}(E)$. Essendo $\|f \cdot g\| \leq\|f\| \cdot\|g\|$ la moltiplicazione in $\not \mathcal{B}(E)$ è continua oltre, che l'addizione e la moltiplicazione scalare, come ci ̀̀ garantito dal fatto che \| $\|$ è una norma.

Sia $\varphi: E \rightarrow \bigcap 3(E)$ l'applicazione di $E$ in $98(E)$ definita ponendo $\varphi(a)=f_{a}$ per ogni $a \in E$ avendo indicato con $f_{a}$ l'elemento di $\not \xi(E)$ definito ponendo $f_{a}(x)=d(a, x)$ per ogni $x \in E$. 
Come ha rilevato $\mathrm{K}$. Kunatowski, da $a \in E, \quad b \in E$ consegue

$$
\|\varphi(a)-\varphi(b)\|=d(a, b) .
$$

Consegue, in primo luogo, che risulta $\varphi(a)=\varphi(b)$ se e solo se è $a=b$, e quindi $\varphi$ è ingettiva.

Inoltre, posto $E^{*}=\varphi(E)$ e detta $\psi$ l'applicazione ridotta di $\varphi$, applicazione di $E$ su $E^{*}$ definita ponendo $\psi(x)=\varphi(x)$ per ogni $x \in E$, e considerato $E^{*}$ quale sottospazio metrico di $\supsetneq(E)$ (con distanza $\|f-g\|$ per due elementi $f$ e $g$ di $\bigcirc(E)$ ), la $\psi$ risulta una isometria di $E$ sopra $E^{*}$.

Sia

$$
A(E)
$$

Ia sottoalgebra chiusa di $\Re(E)$ generata di $E^{*}$ cioè l'aderenza nello spazio metrico $\mathcal{Y}(E)$ della sottoalgebra di $\mathcal{B}(E)$ generata da $E^{*}$ nel senso precisato nel n. 3.

Ovviamente $A(E)$ è una sottoalgebra di BANACH di $\mathscr{B}(E)$.

Sia $B$ una parte ovunque densa di $E$ e sia $B^{*}=\psi(B)$. Per quanto si è riconosciuto nel n. 3, esiste una parte $C$ di $A(E)$ che ha lo stesso numero cardinale di $B^{*}$ ciò̀ di $B$. Dunque

LEMMa 3. - Supponiamo che E sia uno spazio metrico e che a sia un numero cardinale infinito. Allora esiste un'algebra di Banach $A(E)$ tale che $E$ sia isometrico ad un sottospazio metrico di $A(E)$ e se $B$ è una parte di $E$ ovunque densa tale che card $(B) \leq \mathfrak{a}$, esiste una parte $C$ di $A(E)$ ovunque densa tale che card $(C) \leq a$.

Se si osserva ora, che detta parte $B$ può esistere, $E$ essendo metrico, se e solo se esiste una base $\left(T_{\lambda}\right)_{\lambda \in L}$ della topologia di $E$ tale che card $(L) \leq \mathfrak{a}$ si conclude :

Lemma 4. - Se E e a sono quelli del lemma 3 e se $\left(T_{\lambda}\right)_{\lambda \in L}$ è una base della topologia di $E$ tale che card $(L) \gtrless \mathfrak{a}$, esiste un'algebra di Banach $A(E)$ tale che $E$ sia isometrico ad un sottospazio metrico di $A(E)$ e, a sua volta, $A(E)$ abbia una base per la sua topologia $\left(U_{\iota}\right)_{\in \in I}$ tale che card $(I) \leq \mathfrak{a}$.

5. - Dopo questi preliminari si può stabilire che:

Prop. 4. - Supponiamo che E, a e sta(E) siano quelli della prop. 3. Allora supposto che E sia separato (=di Hausdorff), esiste una famiglia $\left(A_{\beta}\right)_{\beta \in B}$ di algebre Banach ciascuna delle quali ha per la sua topologia una base $\left(T_{\beta \lambda} h_{\lambda \in L_{\beta}}\right.$ tale che card $\left(L_{\beta}\right) \leq \mathfrak{a}$ e tale che lo spazio uniforme $\left(E\right.$, $\left.\mathcal{L}_{a}(E)\right)$ sia isomorfo ad un sottospazio uniforme dello spazio uniforme prodotto

$$
\prod_{\beta \in B} A_{\beta}
$$


(munito della strultura uuiforme prodolto delle strutture uniformi metrizzabili di cui ciascun $A_{\beta}$ è munito canonicamente).

Dim. - L'asserto è ovvia conseguenza della prop. 3 e del lemma 4.

Sussiste ora la proposizione seguente che caratterizza la $\mathcal{A}_{a}$-completezza:

Prop. 5. - Se E, a e $\mathscr{A}_{\mathfrak{a}(E)}(E)$ so quelli della prop. 4 e se $E$ è separato, le seguenti proposizioni sono equivalenti:

a) $E$ è $\mathscr{A}_{\mathfrak{a}}$-complelo (cioè lo spazio uniforme $\left(E, \mathcal{A}_{\mathfrak{a}}(E)\right)$ è completo),

b) esiste una famiglia $\left(A_{\beta}\right)_{k \in B}$ di algebre di Banach, ciascuna delle quali $A_{\beta}$ ha una base $\left(T_{\beta \lambda}\right)_{\lambda \in L_{\beta}}$ della sua topologia tale che card $\left(L_{\beta}\right) \leq \mathfrak{a}$ e posto

$$
P=\prod_{\beta \in B} A_{\rho}
$$

$E$ è omeomorfo ad un sottospazio chiuso $E^{*}$ dello spazio prodotto $P$.

Diм. - a) implica b). Supposta vera la a) supponiamo che $\left(A_{\beta}\right)_{\beta \in B}$ sia una famiglia di algebre di BANAOH prevista dalla prop. 4 e $P$ sia definito da (1). Per la prop. 4 esiste un sottospazio aniforme $E^{*}$ di $P$, munito della struttura uniforme prevista nella prop. 4 , tale che $E$ munito di $\mathscr{A}(\mathbb{a}(E)$ sia isomorfo ad $E^{*}$. Poichè $\left(E, \mathscr{A}_{\mathfrak{a}}(E)\right)$ è completo, $E^{*}$, quale sottospazio uniforme, è completo e, poichè $P$ è separato, $E^{*}$ è chiuso in $P$. Da ciò consegue la b).

b) implica a). Sia vera la b). In forza della osservazione 6 al lemma 1 ciascun $A_{\beta}$ è sta-completo e quindi $P$, in forza della prop. 5, n. 2 di [4], è

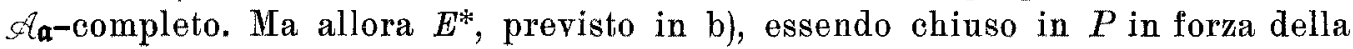
prop. 3 , n. 2 di [4], è $\mathscr{A}_{a}$-completo ed allora $E$ è $\mathscr{A l}_{a}$-completo (osservazione 8 al lemma 1$)$.

Ossenvazione. - E' noto che ogni spazio metrizzabile dotato di base numerabile e, più in generale, ogni spazio di LINDELöF regolare, risulta un $Q$-spazio secondo E. HewrTT (real-compatto secondo [9]). Inoltre il prodotto di una qualunque famiglia di $Q$-spazii è un $Q$-spazio e ogni sottospazio chiaro di un $Q$-spazio è un $Q$-spazio. Allora la prop. 5, per $\mathfrak{a}=\operatorname{card}(\boldsymbol{N})$, dimostra che uno spazio uniformizzabile separato risulta $\mathscr{A}_{\mathfrak{a}}$-completo se e solo se è un $Q$-spazio, (cfr. [4] \& 2, prop. 2) a causa del teor. 11.12 di [9].

Si conclude il presente $\S$ con la proposizione:

Prop. - Supponiamo che $T$ sia uno spazio uniformizabile separato e che E sia un sottospazio di T ovunque denso. Supponiamo, inoltre, che a sia un numero cardinale infinito e che $v_{\mathfrak{a}}(T)$ e $v_{\mathfrak{a}}(E)$ siano gli $\mathcal{G}_{\mathfrak{a}}$-completamenti di $T$ $e$ di $E$ (def. 1). Allora le seguenti proposizioni sono equivalenti:

a) se $A$ è un'algebra di Banach avente una base $\left(T_{2}\right)_{2 \in L}$ per la sua sua topologia tale che card $(L) \leq \mathfrak{a}$ e se $f: E \rightarrow A$ è un' applicazione continua 
di $E$ in $A$ esiste un'applicazione continua $\bar{f}: T \rightarrow A$ dell' intero $T$ in $A$ la cui restrizione a $E$ sia $f$ :

b) se $X$ è un qualunque spazio uniformizzabile separato e Aa-completo e se $f: E \rightarrow X$ è un'applicazione continua di $E$ in $X$ esiste un' applicazione continua $\bar{f}: T \rightarrow X$ dell' intero $T$ in $A$ la cui restrizione ad $E$ sia $f$.

c) risulta $v_{\mathfrak{a}}(T)=v_{\mathfrak{a}}(E)$ (topologicamente).

d) risula $E \subset T \subset v_{\mathfrak{a}}(E)$ (ingettivamente).

Dimostriamo l'asserto stabilendo che a) $\Rightarrow b) \Rightarrow c) \Rightarrow d) \Rightarrow b$ ) $\Rightarrow a$ ).

a) implica b). Supponiamo che $X$ sia quello previsto da b) e supponiamo che $\left(A_{\beta}\right)_{\beta \in B}$ sia una famiglia di algebre di BANACH la cui esistenza è assicurata dalla $b$ ) della prop. 5 con tutte le condizioni ivi dichiarate. sostituende $E$ con $X$. Possiamo identificare, in modo del tutto lecito, $X$ con $X^{*}$ previsto in detta b) prop. 5 riferita ad $X$ pertanto la $f$ può essere riguardata un'applicazione continua di $E$ in $P$.

Per ogni $: \in I$ sia $p r_{\beta}$ la $\beta$-esima proiezione di $P$ (su $A_{\beta}$ ) e poniamo $f_{\beta}=p r_{\beta} \circ f$. La $f_{\beta}$ è un'applicazione continua di $E$ in $A_{\beta}$ : in forza di a), detta $j_{E}$ l'ingezione canonica di $E$ in $T$. esiste un'applicazione continua $g_{\beta}$ di $T$ in $A_{\beta}$ la cui restrizione ad $E$ sia $f_{\beta}$ ciò̀ sia tale che

$$
g_{\beta} \circ j_{E}=p r_{\beta} \circ f
$$

Sia $g=\left(g_{\beta}\right)_{\beta \in B}$ ciò̀ sia $g$ l'applicazione di $T$ in $P$ tale che per ogni $x \in T$ sia $g(x)=\left(g_{\beta}(x)\right)_{B \in B}$. Poichè ogni $g_{\beta}$ è continua anche $g$ è una applicazione continua di $T$ in $P$. A causa della (1) si ha $g \circ j_{E}=f$.

Inoltre, essendo $g(E)=g\left(j_{E}(E)\right)=g \circ j_{E}(E)=f(E)$ risulta

$$
g(T)=g\left(\bar{E}^{(T)}\right) \subset g(\bar{E})^{(P)}=f\left(\bar{E}^{(P)} \subset \bar{X}^{(P)}=X .\right.
$$

Pertanto la $g$ definisce un'applicazione continua $\bar{f}$ di $T$ in $X$ al modo seguente: per ogni $x \in T$ risulta $\bar{f} x)=g(x)$. Evidentemente $\bar{f}_{\circ} j_{E}=f$ e da ciò segue la $b$ ).

b) implica c). Sia vera la b) e sia $f: E \rightarrow X$ una qualunque appli. cazione continua di $E$ in uno spazio uniformizzabile separato ed SAa-com. pleto $X$. Per b) la $f$ si può estendere in una applicazione continua $g$ di $T$ in $X$ e, considerato $T$ come (ingettivamente) immerso quale sottospazio ovunque denso di $v_{\mathfrak{a}}(T)$, per la osservazione alla prop. 1, esiste una esten. sione cotinua $\bar{f}$ di $g$ a $\cup_{\mathfrak{a}}(T)$. Poichè $E$ è ovunque denso in $T$, esso è anche

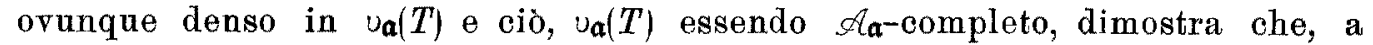
meno di un omeomorfismo, risulta $v_{\mathfrak{a}}(T)=v_{\mathfrak{a}}(E)$ (efr. ancora prop. 1)

c) implica d). È ovvio, in forza della prop. c) e del fatto che è $E \subset T$.

d) implica b). È ovvio, in forza della prop. 1.

b) implica a). E ovvia per la osservazione 6 al lemma 1. 


\section{§ 2. - Filtri $\ell$-inviluppati}

La seguente definizione ha origine da P. SAMvex [11].

DEF. 1. - Se $(E, \mathscr{Q})$ è uno spazio uniforme, una base di filtro $\mathfrak{B}$ su $E$ si dice $\ell$-inviluppata se per ogni $B \in \mathfrak{B}$ esistono un $B^{\prime} \in \mathfrak{B}$ ed una adiacenza $V$ $d i(E, \mathscr{U})$ tali che $V\left(B^{\prime}\right) \subset B$.

Osservazione 1. - La precedente definizione si applica ai filtri che sono, come ò noto, particolari basi di filtro.

OSSERvazIONE 2. - Ogni filtro $U$-inviluppato ha una base formata da insiemi chiusi ed una base formata da insiemi aperti.

OSSERvazione 3. - Se $\mathscr{U}^{\prime}$ è una nlteriore struttura uniforme su $E$ più fine di $U$ ogni base di filtro $U$-inviluppata è anche $U^{\prime}$ inviluppata.

Prop. 1 - Se $(E, \mathcal{Q})$ è uno spazio uniforme $e$ se $\mathfrak{F} \dot{e}$ un filtro su $E$ le seguenti propozizioni sono equivalenti :

a) $\mathfrak{F} \grave{e} \mathscr{U}$-inviluppato (def. 1)

b) ogni base di $\mathfrak{F}$ è K-inviluppata

c) esiste una base $\mathscr{C}$-inviluppata per $\mathfrak{F}$.

Drm. - Che $a) \Rightarrow b$ ) $\Rightarrow$ c) è ovvio.

c) implica a). Sia $\mathfrak{B}$ una base $\ell$-inviluppata di $\mathfrak{F}$ e sia $F \in \mathfrak{F}$. Esiste un $B \in \mathfrak{B}$ tale che $B \subset F$ ed esistono un $B^{\prime} \in \mathfrak{B}$ de un'adiacenza $V$ di $(E, \chi \ell$ tali che $V\left(B^{\prime}\right) \subset B$. Ciò, essendo $\mathfrak{B} \subset \mathfrak{F}$ dimostra la a).

Un esempio di filtro $\mathfrak{C}$-inviluppato è fornito dalla seguente

Prop. 2. - Se $(E, \mathcal{Q})$ è uno spazio uniforme, per ogni $x \in E$, il filtro $\mathfrak{B}(x)$ degli intorni di $x$ è Q-inviluppato.

DLM. - Sia $W \in \mathfrak{B}(x)$ : esiste un'adiacenza $V$ di $(E, \mathscr{U})$ tale che $V \circ V(x) \subset W$. Poichè. $V(x)$ è un intorno di $x$ e risulta $V(V(x))=V \circ V(x) \subset W$ la tesi è vera.

Ulteriori esempii di filtri $\ell$-inviluppati sono forniti dalla seguente proposizione :

Prop. 3. - Se $(E, 9)$ è uno spazio uniforme e se $\mathfrak{F}$ è un filtro su E, esiste un filtro $U$-inviluppato $\Im$ su $E$ (def. 1) che è meno fine di $\mathfrak{F}$ ed è tale che, supposto che $\subseteq$ sia un sistema fondamentale di adiacenze di $(E, \mathcal{Z})$ e che $\mathfrak{B}$ sia base di $\mathfrak{F}$, l'insieme $\mathfrak{B}_{\mathfrak{S}}$ delle parti $B$ di $E$ della forma $B=V(A)$ con $V \in \mathfrak{S}$ ed $A \in \mathfrak{B}$, ̀̀ base di $\mathfrak{\Im}_{\imath t}$.

Drm. - Che $\mathfrak{B}_{\subseteq}$ sia una base di filtro consegue dal fatto ohe $\subseteq$ e $\mathfrak{B}$ sono basi di filtro. 
Riconosciamo che $\mathfrak{B}_{\mathfrak{S}}$ è $\ell$-inviluppato. Infatti se è $B \in \mathfrak{B}_{\mathbb{S}}$ esistono $V \in \mathbb{S}$ ed $A \in g$ tali che $B=V(A)$. Poichè $\subseteq$ è un sistema fondamentale di adia. cenze per $(E, \mathscr{C})$ esiste un $W \in \subseteq$ tale che $W \circ W \subset V$. Posto $B^{\prime}=W(A)$ risulta $B^{\prime} \in \mathfrak{B}_{\subseteq}$ e $W\left(B^{\prime}\right) \subset B$ il che, essendo $W \in \mathfrak{S}$, dimostra che la base $\mathfrak{B}_{\mathfrak{S}} \grave{e}$ U-inviluppata.

Sia $\mathfrak{A}$ il fil tro delle adiacenze di $(E, \nearrow C)$ (del quale $\mathfrak{S}$, per ipotesi, è una base). Se in quanto sopra si assume $\mathfrak{B}=\mathfrak{F}$ ed $\mathfrak{S}=\mathfrak{A}$ risulta che $\mathfrak{F}_{\mathfrak{Y}}$ è una base di filtro $\ell$-inviluppata (osservazione 1 alla def. 1) ed il filtro $\mathfrak{F}_{\mathfrak{C}}$ da essa generato e, del pari, $\ell$-inviluppato.

Inoltre ogni $\mathfrak{B}_{\mathfrak{S}}$ è base di $\mathfrak{F}_{\mathscr{U}}$ ed $\mathfrak{F}_{\mathcal{X}}$ è meno fine di $\mathfrak{F}$.

Dopo ciò è giustificata la definizione che avrà ufficio essenziale, in ap. presso.

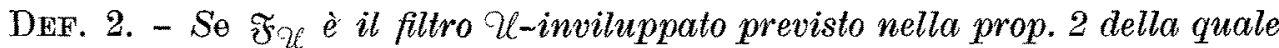
conseviamo notazioni e definizioni, ad $\mathfrak{F}_{\mathfrak{C}}$ si attribuisce il nome di U-inviluppo di $\mathfrak{F}$ oppure di filtro $\ell$-inviluppato generato da $\mathfrak{F}$.

Osservazione 1. - Se $U$ e $U^{\prime}$ sono strutture uniformi su $E$ ed $\mathscr{U}$ è meno fine di $\mathscr{U}^{\prime}$ e se $\mathfrak{F}$ e $\mathfrak{F}^{\prime}$ sono filtri su $E^{\prime}$, l' $\mathscr{\ell}$-inviluppo $\mathfrak{F}_{\ell}$ di $\mathfrak{F}$ è meno fine dell' $\ell^{\prime}$-inviluppo di $\mathfrak{F}^{\prime}$.

OSSERVAZIONE 2. - Se $\mathfrak{F}$ gode della proprietà dell'intersezione numerabile (cfr. il seguente $\S 3$. def. 1) altrettanto accade ad $\mathfrak{Z}_{\mathfrak{K}}$.

Come ò ovvio:

Prop. 4. - Se (E, $\ell)$ è uno spazio uniforme, se $\mathfrak{F}$ è un filtro sull' insieme $E$ se $\mathfrak{F}$ è il fittro U-inviluppato generato da $\mathfrak{F}$ (def. 2) le seguenti proposi. zioni sono equivalenti;

a) $\mathfrak{F}$ è $\nVdash$-inviluppato.

b) risulta $\mathfrak{F}=\mathfrak{F}_{\mathscr{C}}$.

DrM. - a) implica b). Dalla def. 2 e dalla prop. 3 consegue $\mathfrak{F}_{\nearrow} \subset \mathfrak{F}$ mentre, poichè $\mathfrak{F}$ è $\ell$ - inviluepato si ha $\widetilde{F} \subset \mathfrak{F}_{\ell}$. Da ciò la b).

b) implica a). E ovvia conseguenza della def. 2 e della prop. 3.

Osservazione. - Se $\mathfrak{F}$ è $\chi$-inviluppato e se $\mathcal{F}^{\prime}$ è un filtro più fine di $\mathfrak{F}$ l'U-inviluppo $\mathfrak{F}^{\prime} \mathscr{X}$ di $\mathfrak{F}^{\prime}$ (def. 2) è più fine di $\mathfrak{F}$. Consegue dalla osserva. zione 1 alla def. 2 e dalla prop. 4.

Di particolare utilità risulterà la

Prop. 5. - Se (E, भ) e uno spazio uniforme. se $\mathfrak{F} \dot{e}$ un filtro su $E$ e se $\mathfrak{F}_{\mathfrak{U}} \grave{e}$ il filtro U-inviluppato generato da $\mathfrak{F}$ (def. 2) le seguenti proposizioni sono equivalenti: 
a) ₹̛̀ un filtro di Cauchy su (E, Ø),

b) $\mathfrak{\mho}_{q}$ è un filtro di Cauchy su $(E, \eta \ell$.

DIM. - a) implica b). Sia $V$ un'adiacenza di $(E, \mathcal{Q})$ e sia $W$ un'adiacenza simmetrica di $(E, \mathcal{U})$ tale che $W_{\circ} W_{\circ} W \subset V$. Se è vera la a) esiste un $F \in \mathfrak{F}$ tale che $F \times F \subset W$ : allora $W(F)$ è piccolo di ordine $W \circ W \circ W$ e quindi di ordine $V$, il che, essendo $W(F) \in \mathfrak{F}_{\nearrow}$ dimostra la b).

b) implica a). É ovvia poichè $\mathfrak{F}_{\mathfrak{Z}}$ è meno fine di $\mathfrak{F}$.

La seguente proposizione ha un notevole interesse tecnico.

Prop. 6. - Se $(E, \mathcal{X})$, $\mathfrak{F}$ ed $\mathfrak{F}_{\ell}$ sono quelli della prop. 5, ogni qual volta

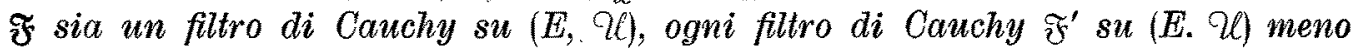
fine di $\mathfrak{F}$ risulta più fine di $\mathfrak{\mho} \mathscr{U}$.

Dim. - Sia $A \in \mathfrak{F}_{\mathscr{X}}$ : eistono $B \in \mathfrak{F}$ ed un'adiacenza $V$ di $(E, \mathscr{\ell})$ tali che $A=V(B)$. Poichè $\widetilde{F}^{\prime} \grave{e}$ un filtro di Cauory su $(E, \eta \ell)$ esiste $F^{\prime} \in \mathscr{F}^{\prime}$ tale che $F^{\prime} \times F^{\prime} \subset V$ : poichè $\mathfrak{F}^{\prime}$ è meno fine di $\mathfrak{F}$, risulta anche $F^{\prime} \in \mathfrak{F}$ e quindi $B \cap F^{\prime \prime} \neq \varnothing$ donde, $F^{\prime}$ essendo piccolo di ordine $V$, consegue $F^{\prime} \subset V(B)=A$. Per un assioma di filtro, è $A \in \mathfrak{F}^{\prime}$ ed $\mathfrak{F}^{\prime}$ è più fine di $\mathfrak{F}_{\mathscr{\ell}}$.

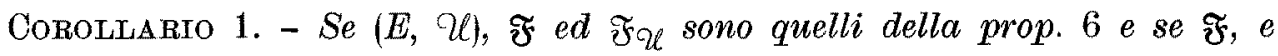

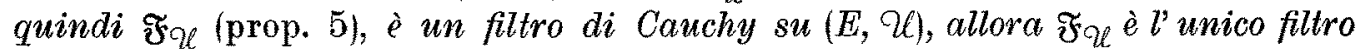
di Cauchy minimale su $E$, meno fine di $\mathfrak{F}$, sull' insieme, ordinato per finezza, dei filtri di Cauchy su $(E, \mathscr{\ell})$.

Drm. - Detto $\mathfrak{F}^{\prime}$ un filtro di CAđoHr su $(E, \mathscr{K})$, se risulta che $\mathfrak{F}^{\prime}$ è meno fine di $\mathfrak{F}_{\mathscr{L}}, \mathfrak{F}^{\prime}$ è meno fine di $\mathfrak{F}$ (def. 2 e prop. 3) e quindi, per la prop. 6, $\mathfrak{F}^{\prime}$ risultando più fine di $\mathfrak{F}$, si ha $\mathfrak{F}=\mathfrak{F}^{\prime}$.

Se, invece, si suppone che $\mathcal{F}^{\prime}$ sia un filtro di CADCHY minimale $\theta$ meno fine di $\mathfrak{F}$, per la prop. 6 , si ha che $\mathfrak{F}^{\prime}$ è più fine di $\mathfrak{F}$ e, per la minimaiità $\mathfrak{F}=\mathfrak{F}^{\prime}$.

Corollario 2. - (H.J. Kowalski [10]). Se (E, $\ell$ ) è uno spazio uniforme se $\mathfrak{F}^{\prime} \grave{e}$ un filtro $\ell$-inviluppato e se $\widetilde{F}^{\prime \prime} \dot{e}$ un filtro di CAUCHY su $(E, \mathscr{Q})$, ogni volta che esista un filtro $\mathfrak{F} s u$ Eiù fine di $\mathfrak{F}^{\prime}$ ed $\mathfrak{F}^{\prime \prime}$, allora $\mathfrak{F}^{\prime} \dot{e}$ meno fine $d i \mathfrak{F}^{\prime \prime}$.

Dim. - Sia $\mathfrak{F}_{\mathscr{U}}$ il filtro $\mathscr{\ell}$-inviluppato su $E$ generato da $\mathfrak{F}$ (def. 2.). Poichè $\mathfrak{F}$ è più fine del filtro di CAUOHY $\mathfrak{F}^{\prime \prime}$ anche $\mathfrak{F}$ è un filtro di CaUchy su $(E, \mathscr{\ell})$ e quindi, per la prop. $6, \mathfrak{F}_{\mathscr{X}}$ è meno fine di $\mathfrak{F}^{\prime \prime}$.

Allora. per l'osservazione alla prop. 4 , $\mathfrak{F}_{\mathscr{C}}$ e più fine di $\mathfrak{F}^{\prime}$. Poichè $\mathfrak{F}_{\mathscr{U}}$ è meno fine di $\mathfrak{F}^{\prime \prime}$ (prop. 6) la tesi ̀̀ dimostrata.

Corollario 3. - Se $(E, \eta)$ è uno spazio uniforme, se $\vec{J}^{\prime}$ è un filtro $\ell-$ -inviluppato su $E$ (def. 1) più fine del filtro di Cauchy $\mathfrak{F}^{\prime \prime}$ su (E, Ul), si ha $\mathfrak{F}^{\prime}=\mathfrak{F}^{\prime \prime}$. 
Dru. - Nel corollario 2 ora può assumersi $\mathfrak{F}=\mathfrak{F}^{\prime} \dot{e}$ quindi $\mathfrak{F}^{\prime} \dot{e}$ meno fine di $\mathfrak{F}^{\prime \prime}$; poichè, per ipotesi, $\mathfrak{F}^{\prime \prime}$ è meno fine di $\mathfrak{F}^{\prime}$, la tesi è vera.

Osservazione. - Se $\mathfrak{F}$ è un filtro di Cadohr su $(E, \mathcal{C})$, sappiamo, per la prop. 4, che anche l' $\ell$-inviluppo $\mathfrak{F}_{\chi}$ di $\mathfrak{F}$ è un filtro di CAUcHY, per cui, se $\mathfrak{F}^{\prime}$ è un filtro $\mathscr{U}$-inviluppato $\mathrm{su}_{4}(E, \mathfrak{Q})$ più fine di $\mathfrak{F}_{\ell}$ si ha $\mathfrak{F}^{\prime}=\mathfrak{F}_{\mathscr{C}}$. Dunque $\mathfrak{\mho}_{\mathscr{U}} \dot{e}$ un filtro $\ell$-inviluppato massimale, cioè un elemento massimale dell' insieme dei filtri $\ell$-inviluppati, ordinato per finezza. Si rilevi che l'esistenza di $\mathfrak{F}_{\mathscr{U}}$ è stabilita senza l'ausilio del lemma di ZonN e, se $(E, \mathscr{U}$ ) non ə̀ compatto esistono filtri $\mathscr{U}$-inviluppati massimali oltre quelli previsti dalla prop. 2.

Del lemma di ZonN, al contrario ei si serve per stabilire l'esistenza di ultrafiltri non banali e di ciò si fa uso nella seguente

Prop. 7. - Supponiamo che $(E, 9)$ sia uno spazio uniforme precompatto

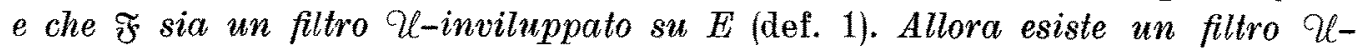
-inviluppato di Cauchy su $(E, \mathscr{U})$ più fine di.

DIм. - Sia $\mathfrak{A}$ un ultrafiltro su $E$ più fine di $\mathfrak{F}$ e sia $\mathfrak{A}_{\mathscr{U}}$ il filtro $\mathscr{\ell - i n - ~}$ viluppato generato da $\mathfrak{A}$ (def. 2). Poichè $\mathfrak{Z}$ è un filtro di Cauchy su $(E, \mathscr{U})$ (stante la precompatezza) $\mathfrak{U}_{\mathcal{U}}$ è del pari un filtro di CAUCHY (prop. 5). Ciò per la osservazione 1 alla def. 2 e per la prop. 4, dimostra che $\mathfrak{A}_{\mathfrak{U}}$ è più fine di ₹.

Osservazione. - Per quanto precisato nella osservazione al corollario 3 , $\mathfrak{A}_{\mathfrak{C}}$ è un filtro $\mathcal{C}$-inviluppato massimale.

Lemma 2. - Supponiamo che $(E, \mathscr{U})$ sia uno spazio uniforme, che $\mathfrak{F}$ sia un filtro di Cauchy su $(E, \mathcal{U})$ e che $A$ e $B$ siano parti di $E$ tali che sia $V(A) \subset B$ per almeno un'adiacenza $V$ di $(E, \vartheta)$. Allora o risulta $B \in \mathfrak{F}$ oppure risulta $\mathrm{C} A \in \mathfrak{F}$.

DIM. - Se per ogni $F \in \preccurlyeq$ risulta $F \cap A \neq \varnothing$, poichè $\mathfrak{F}$ è un filtro di CAdOHY esiste un $F \in \mathfrak{F}$ tale che $F \times F \subset V$ e quindi si ha $F \subset V(A) \subset B$. Se, invece, esiste $F \in \mathfrak{F}$ tale che $F \cap A=\varnothing$ si ha $F \subset \mathbf{Q} A$ e quindi $\mathbf{C} A \in \mathfrak{F}$.

Si dovrì utilizzare un risultato noto, relativo alla teoria generale delle strutture uniformi di spazio precompatto.

LeMma 3. - Se $(E, \mathcal{U})$ è uno spazio uniforme esiste una (ed una sola) slruttura uniforme $\mathscr{A}(\mathcal{U})$ su $E$, compatibile con la topologia dedotta da $\mathscr{U}$ su $E$ e che risulta la più fine delle strutture uniformi di spazio precompatto su $E$ meno fini di $\mathcal{~}$. Inoltre, una parte $V$ di $E \times E$ è una adiacenza di $(E, \mathscr{A}(\mathcal{Q}))$ se e solo se esistono un ricovrimento chiuso $\left(F_{k}\right)_{0 \leq k \leq n}$ ed un ricovrimento aperto $\left(G_{k}\right)_{0 \leq k \leq n} d i E$ ed una adiacenza $W$ di $(E, \mathcal{U})$ tali che risulti, per ogni $k=0, \ldots, n$

$$
W\left(F_{k}\right) \subset G_{k}
$$


e si abbia:

$$
\bigcup_{k=0}^{n}\left(G_{k} \times G_{k}\right) \subset V
$$

DrM. - Consegue dal coroll. 1 alla prop. 3 e dal lemma 3 di [2] $\$ 2$.

Osservazione. - Per brevità, aniformandoci alla def. 1 del $\$ 2$ di [2], la $\mathscr{A}(\mathcal{C l})$ sarà denominata "la struttura uniforme associata ad $\mathcal{U}$ 》cioè assn. miamo la seguente definizione:

DEF. 3 - Se $(E, \mathcal{Q})$ ed $\mathscr{A}(\mathcal{Q})$ sono quelli previsti nel lemma 3 , $q(\neg)$ dicesi la struttura uniforme associata ad $\ell$.

Lemma 4. - Se $(E, \mathcal{X})$ è uno spazio uniforme, se $\mathcal{A}(\mathcal{Q})$ è la struttura uniforme associata ad $\mathcal{U}$ (def. 3) e se $A$ e $B$ sono parti di $E$ tali che per un'adiacenza $V$ di $(E, \mathcal{U})$ sia $V(A) \subset B$, allora esiste un'adiacenza $W$ di $(E, \mathscr{A}(\mathcal{l l}))$ tale che $W(\overrightarrow{A)} \subset B$.

Dim. - La tesi consegue dal lemma $1, \S 2$, dalla def. $2, \S 2$ e dalla prop. $2, \S 1$ di $[3]$.

Prop. 8 - Se $(E, \mathcal{U})$ e $\mathscr{S}(\mathcal{U})$ sono quelli del lemma 4 e se $\mathfrak{F}$ è un filtro su $E$, le seguenti sono equivalenti :

a) $\mathfrak{F} \grave{e}$ U-inviluppato (def. 1 )

b) $\mathfrak{F}$ è $\mathscr{A}(\mathcal{Q})$-inviluppato.

DrM. - a) implica b). Consegue dalla def. 1 e dal lemma 4.

b) implica a). Consegue dal fatto che $\mathscr{A}(\mathscr{O})$ è meno fine di $\mathscr{U}$.

La seguente proposizione ha ufficio fondamentale in seguito.

Prop. 9. - Se $(E, \mathscr{U})$ e $\mathscr{A}(\mathcal{U})$ sono quelli del lemma 4 e se $\mathfrak{F} \dot{e}$ un filtro U-inviluppato su $(E, \mathscr{\ell})$ (def. 1) le seguenti proposiaioni sono equivalenti:

a) $\mathfrak{F}$ è un filtro di Cauchy su $(E, \mathscr{q}(20))$,

b) se $A$ e $B$ sono parti di $E$ tali che per un'adiacenza $V$ di $(E$, $\mathcal{A}(\mathcal{Q}(\ell))$ sia $V(A) \subset B$, allora risulta $B \in \mathfrak{F}$ oppure $\mathrm{Q} A \in \mathfrak{F}$,

c) se $\left(A_{k}\right)_{0 \leq k \leq n} e\left(B_{k}\right)_{0 \leq k \leq n}$ sono famiglie finite di parti di $E$, se esiste un' adiacenza $V$ di $(E, \mathscr{A}(\mathcal{l}))$ tale che, per ogni $k=0,1, \ldots, n$, sia $V\left(A_{k}\right) \subset B_{k}$ e se risulta $\bigcup_{k=0}^{n} A_{k} \in \mathfrak{F}$, allora esiste un $k=0,1, \ldots, n$ tale che risulti $B_{k} \in \mathfrak{F}$.

d) Se $\left(A_{k}\right)_{0 \leq k \leq n}$ e $\left(B_{k}\right)_{0 \leq k \leq n}$ sono ricovrimenti di $E$ ed esiste un' adiacenza $V$ di $\left(E, \mathcal{A}\left(\mathcal{l}(\mathrm{l})\right.\right.$ tale che per ogni $k=0,1, \ldots, n$ risulli $V\left(A_{k}\right) \subset B_{k}$, allora esiste un $k=0,1, \ldots, n$ tale che sia $B_{k} \in \mathfrak{F}$. 
DrM. - a) implica b). Consegue dal lemma 2.

b) implica c). Se per ogni $k=0,1, \ldots, n$ fosse $\mathrm{C} A_{k} \in \mathfrak{F}$, per un assioma di filtro risulterebbe $\mathfrak{G}\left(\cup_{k=0}^{n} A_{k}\right)=\left(\bigcap_{k=0}^{n}\left(\mathbb{C} A_{k}\right)\right) \in \mathfrak{F}$, contro l'ipotesi $\bigcup_{k=0}^{n} A_{k} \in \mathfrak{F}$. Dunque è $B_{k} \in \mathfrak{F}$ per almeno un $k$.

c) implica d). Infatti è $\bigcup_{k=0}^{n} A_{k}=E \in \mathfrak{F}$.

d) implica a). Consegue dalla def. 3 e dal lemma 3.

Osservazione. - In forza del lemma 4, in b), c) e d), s(l( $)$ può essere sostituito da $\mathscr{U}$.

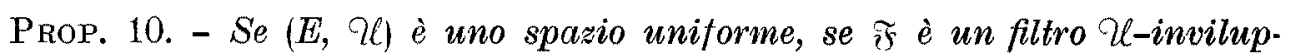
pato e se $\grave{e} x \in E$, le seguenti proposizioni sono equivalenti.

a) risulta $x \in \bigcap_{F \in \widetilde{F}} \bar{F}$,

b) risulta $x \in \cap F$. $\overrightarrow{F \in F}$

DrM. - a) implica b). Se è vera la a) in forza della def. 1 e del lemma 4, poichè $\chi$ è meno fine di $\mathscr{A ( \chi \ell ) , ~ s i ~ h a ~ l a ~ b ) . ~}$

b) implica a). É ovvia.

DEF. 4 - Se $(E$, Q ¿ è uno spazio uniforme chiamasi filtro U-inviluppato (def. 1) massimale ogni elemento massimale dell' insieme dei filtri $\mathscr{C}$-inviluppati su E, ordinato per «finezza».

OSSERVAZIONE. - Di questa nozione ci siamo già serviti nella osservazione al corollario 3 della prop. 6 . Tale osservazione. ha fornito esempi rilevanti di filtri $\mathscr{\ell}$-inviluppati massimali.

Prop. 11 - Se $(E, \mathcal{U})$ è uno spazio uniforme, se $\mathfrak{F}$ è un filtro $\nVdash$-inviluppato (def. 1) su $E$ e se $G(\mathcal{U}$ ) è la struttura uniforme associata ad $\mathcal{U}$ (def. 3), le seguenti proposizioni sono equivalenti:

a) $\mathfrak{F}$ è un filtro di Cauchy su $(E, \mathcal{A}(\mathcal{Q l}))$,

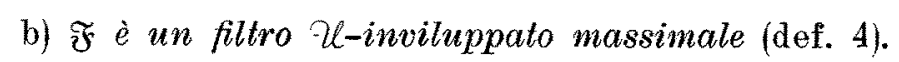

DrM. - a) implica b). Sia $\widetilde{F}^{\prime}$ un filtro $\ell$-inviluppato più fine di $\mathfrak{F}$. A causa della prop. 8 , $\widetilde{F} \cdot e d \widetilde{F}^{\prime}$ sono $\mathscr{A}(\mathcal{C} \ell$-inviluppati e quindi, in forza del corollario 3 alla prop. 6 , da a) consegue b).

b) implica a). Supponiamo che $\mathfrak{F}$ sia un filtro $\ell$-inviluppato massimale. Ancora per la prop. 8 , $\mathfrak{\mho}$ è $s(2 \ell)$-inviluppato e quindi per la precompattezza 
di $(E, \mathscr{A}(\mathcal{C}))$ esiste, per la prop. 7, un filtro $\mathscr{A}(\mathcal{C})$-inviluppato di CadoHY su $(E, s \mathcal{A}(\mathcal{C}))$ più fine di $\mathfrak{F}$ che, per la massimalità di $\mathfrak{F}$, coincide con $\mathfrak{F}$.

Chiarito in questo modo la struttura dei filtri $\ell$-inviluppati massimali, riconosciamo che:

Prop. 12 - Se $(E, \mathcal{U}) \grave{e}$ uno spazio uniforme, se $\mathfrak{\xi} \grave{e}$ un filtro $U$-inviluppato massimale e se è $x \in E$, le seguenti proposizioni sono equivalenti:

a) risulta $x \in \bigcap_{F \in \Im} \bar{F}$,

b) risulta $x \in \cap F$. $F \in \Im$

c) $\mathfrak{F}$ converge verso $x$.

Dim. - a) implica b). È ovvia conseguenza della prop. 10.

b) implica c). Poichè $\mathfrak{F}$ è $U$-inviluppato da b) consegue che ogni $F \in \mathfrak{F}$ $\grave{e}$ intorno di $x$ e quindi $\mathfrak{F}$ è meno fine del filtro $U$-inviluppato (prop. 2) $\mathfrak{B}(x)$ degli intorni di $x$ e quindi per la def. 4 è $\mathfrak{F}=\mathfrak{B}(x)$.

c) implica a). 亡̀ ovvia.

Osservazione. - Se lo spazio uniforme $(E, \mathscr{\ell})$ di cui sopra è separato, se $\mathfrak{F}$ ha intersezione non vuota esso converge verso ciascun elemento dell'intersezione e quindi, a causa dell'assioma di HADSDORFF, la sua intersezione è formata da un unico punto $x(\mathfrak{F})$ di $E$. Inoltre due filtri $\ell$-inviluppati massimali non possono avere uno stesso punto di $E$ come loro intersezione senza coincidere (come consegue dalla b) $\Leftrightarrow$ ) e dalla massimalità). Dunque la $\mathfrak{F} \rightarrow x(\mathfrak{F})$ è un' applicazione bigettiva dell'insieme dei filtri $\mathscr{U}$-inviluppati massimali, aventi intersezione non vuota, sull'insieme $E$.

Si osservi anche che:

Prop. 13 - Se $(E, \mathcal{U})$ è uno spazio uniforme e se $\mathfrak{F}$ è un filtro $\ell-i n v i-$ luppato (def. 1) le seguenti proposizioni sono equivalenti:

a) $\mathfrak{F}$ converge,

b) $\mathfrak{F}$ è un filtro U-inviluppato massimale (def. 4) e risulta non vuota la sua intersezione.

DrM. - La necessità è stata chiarita da quanto sopra e la sufficienza consegue dalla prop. 12.

Prop. 14 - Se $(E, \mathcal{Q})$ ed $\mathfrak{F}$ sono quelli della prop. 13 , esiste un filtro U-inviluppato massimale più fine $d i$ F (def. 4). 
Drm. - Consegue dalle propp. 7, 8 e 11 .

Osservazione. - Si noterà che la proposizione stabilita consegue dalla prop. 7 ed in conseguenza del lemma di ZorN e quindi dall'assioma della scelta. Cio va posto in contrasto con l'osservazione al corollario 3 della prop. 6.

La precompattezza di uno spazio uniforme può essere caratterizzato mediante filtri $\mathscr{\ell}$-inviluppati massimali. A tal fine premettiamo un lemma del quale si farà uso anche altrove.

LFmмa 5. - Se V è una parte simmetrica contenente la diagonale dell' insieme $E \times E$ prodotto dall' insieme $E$ per se stesso, esiste una parte. $A d i E$ tale che da $x \in A, y \in A$ e $x \neq y$ consegua $y \in \mathcal{G} V(x)$ e tale che sia $E=\bigcup_{x \in A} V(x)$.

Drm. - Detto $\mathscr{Q}$ l'insieme delle parti $X$ di $E$ tali che da $x \in X, y \in Y$ $x \neq y$ consegua $y \in C V(x)$, si riconosce che $\mathfrak{A}$ ordinato per inclusione è induttivo e quindi, per il lemma di ZorN, esiste un suo elemento massimale per inclusione $A$ che è quello previsto dalla tesi.

Prop. 15. - Se $(E, \mathcal{Q})$ è uno spazio uniforme non precompatto esistono una parte infinita $A$ di $E$ ed un'adiacenza $V$ di $(E, \mathcal{U})$ tali che da $x \in A$, $y \in A$ e $x \neq y$ consegua $y \in G V(x)$ ed inoltre risulti $E=\bigcup_{x \in A} V(x)$.

Dim. - Se $(E, \mathscr{C})$ non è precompatto esiste un'adiacenza $W$ di $(E, \mathscr{C})$ tale che $E$ non sia riunione di alcuna famiglia finita di sue parti piccole di ordine $W$. Sia $V$ un'adiacenza simmetrica di $(E, \mathscr{\ell})$ tale che $V \circ V \subset W$. Costituita, in relazione a questo $V$, la parte $A$ di $E$ prevista nel lemma 5, ogni $V(a)$, per $a \in A$, è piccolo di ordine $W$ ed essendo $E=\bigcup_{a \in A} \nabla(a)$, se $A$ fosse finita si contradirebbe la definizione di $W$.

Osservazione. - Consegue che se $(E, \mathscr{Z})$ è numerabilmente compatto esso è anche precompatto per eiò, se in più, si assume che $\ell$ sia metrizza. bile, lo spazio $E$ ha base numerabile $e$ quindi (\$ 1 osservazioni 5 ed 1 al lemma 1), $F$ è uno spazio di Lindelöf. Pertanto essendo numerabilmente compatto, $E$ è compatto: ciò è ben noto.

Sussiste la seguente caratterizzazione della precompattezza, già dimostrata, per altra via, in [1] n. 1, teor. 2, pag. 100.

PRop. 16. - Se $(E, \mathscr{U})$ è uno spazio uniforme le seguenti proposizioni sono equivalenti:

a) $(E, \mathfrak{U})$ è precompatto.

b) Ogni filtro $\ell$-inviluppato massimale (def. 4) è un filtro di Cauchy $s u(E, \mathcal{Q})$. 


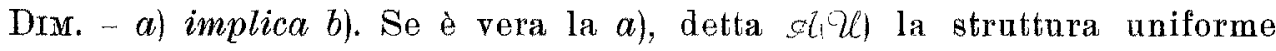

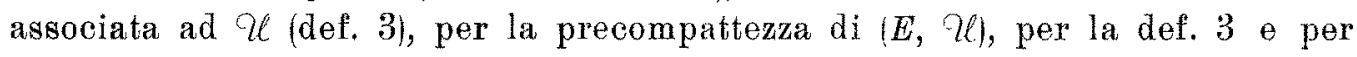
il lemma 3 , risulta $U=\mathscr{A}(\mathcal{U})$ e da ciò, in forza della prop. 11 , consegue la attuale b).

b) implica a) Sia vera la b) e, ragionando per assurdo, supponiamo che $(E, \mathscr{U})$ non sia precompatto. Allora, in forza della prop. 13, esistono una parte infinita $A$ di $E$ ed un'adiacenza $V$ di $(E, \mathscr{\ell})$ tale, che $x \in A, y \in A$ e $x \neq y$ consegua $y \in \mathbf{G} V(x)$.

Sia $\mathfrak{A}$ l'insieme delle pârti $X$ di $E$ della forma $A \cap(G B)$ dove $B$ è una parte finita di $A$ : è subito visto che $\mathfrak{A}$ è base di un filtro $\mathfrak{B}$ e denotiamo

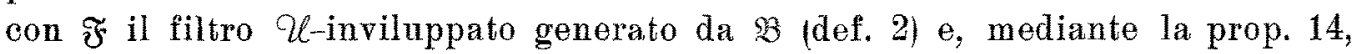
consideriamo un filtro $\mathscr{U}$-inviluppato massimale $\mathfrak{M}$ (def. 4) più fine di $\mathfrak{F}$.

Sia $W$ un'adiacenza aperta simmetrica di $(E, \mathscr{U})$ tale che $W \circ W_{\circ} W_{\circ} \subset V$.

Per $b), \mathfrak{M}$ e un filtro di CaUCHY su $(E, \mathscr{U})$ e quindi esiste un $M \in \mathfrak{M}$ piccolo di ordine $W$ (cioè $M \times M \subset W$ ). Evidentemente, per ogni $B \in \mathfrak{A}$, risultando $\mathfrak{A} \subset \mathfrak{B}$, si ha $W(B) \in \mathfrak{F} \subset \mathfrak{M}$ e quindi $W(B) \cap M \neq \varnothing$.

Poichè risulta $W(B)=\bigcup_{b \in B} W(b)$, esiste $a \in B$ tale che $W(a) \cap M \neq \varnothing$.

Ora sia $B^{\prime}=B \cap(\mathbb{C}\{a\})$. Poichè è $B^{\prime} \in \mathfrak{Q}$, come prima si deduce che esiste $b \in B^{\prime}$ tale che $W(b) \cap M \neq \varnothing$. Poichè $M$ è piccolo di ordine $W$, dalla simmetria di $W$, consegue $(a, b) \in W \circ W \circ W \subset V$ e quindi $b \in V(a)$. D'altra parte ̀̀ $a \in A, b \in A$ e $a \neq b$ : per definizione di $A$ consegue $b \in C V(a)$ ciò che è escluso.

Dunque vera è la $a$ ).

Indichiamo ora una caratterizzazione degli spazii uniformi compatti. A tal fine, premettiamo la

Prop. 17. - Se $(E, \eta)$ è uno spazio uniforme, se $\mathfrak{F} \grave{e}$ un filtro su $E$ e se $\mathfrak{F}$ è il filtro U-inviluppato generato da ₹ l'aderenza di $\mathfrak{F}$ è identica a quella $d i \mathfrak{q} \cdot$

Drm. - Poichè $\mathfrak{F}_{\mathcal{Z}}$ è meno fine di $\mathfrak{F}$, l'aderenza $A$ di $\mathfrak{F}$ è contenuta nell'aderenza $B$ di $\widetilde{F}_{\Upsilon C}$. Dimostriamo che, reciprocamente, è $B \subset A$. Notiamo che, in forza della prop. 12, si ha $B=\cap H$. Sia dunque $x \in B$ e, ragionando per

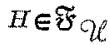

assurdo supponiamo $x \in \mathbf{B} A$ e quindi che esista un'adiacenza $V$, che possiamo supporre simmetrica, di $(E, \mathscr{U})$ tale che $V(x) \cap F=\varnothing$ per almeno un $F \in \mathfrak{F}$. Si ha $x \in V(F)$ e quindi esiste $y \in F$ tale che $x \in V(y)$, donde, per la simmetria di $V$, risulta $y \in V(x)$ e poi $y \in V(x) \cap F \neq \varnothing$. Dunque ̀̀ $B \subset A$.

Dopo cio la notevole

Phop. 18. - Se $(E, U)$ è uno spazio uniforme le seguenti proposizioni sono equivalenti:

a) $(E, \mathcal{\ell})$ è compatto, 
b) $(E, Z)$ è precompatto e completo,

c) ogni filtro C-inviluppato massimale (def 4) ha intersezione non vuota,

d) ogni filtro q-inviluppato (def. 1) ha intersezione non vuota.

DrM. - a) implica b). 亡̀ ovvia.

b) implica $c$ ). Se è vera la $b$ ), per la prop. 16, ogni filtro $U$-inviluppato massimale è di CAUrHY, per la completezza, converge e, per la prop. 13, ha intersezione non vuota.

c) implica d). Consegue dalla prop. 14 se si suppone vera la c).

d) implica a). Se $\widetilde{F}$ è un qualunque filtro su $E$, se è vera la $d$ ), il filtro $\mathscr{U}$-inviluppato generato da $\mathscr{F}$ (def, 1) ha intersezione non vuota e quindi anche $\mathfrak{F}$, essendo vera la prop. 12.

Prop. 19. - Se $f$ è un'applicazione uniformemente continua dello spazio uniforme $(E, \mathscr{U})$ sullo spazio uniforme $\left(E^{\prime}, \mathscr{U}^{\prime}\right)(f(E)=E)$ e se $\mathfrak{F}^{\prime}$ è un filtro $\mathscr{U}^{\prime}$-inviluppato su $\left(E^{\prime}, \mathfrak{U}^{\prime}\right)$ la sua immagine reciproca $f^{-1}\left(\mathfrak{F}^{\prime}\right)$ è base di un filtro $\mathscr{\mho}$-inviluppato su $(E, \mathcal{U})$.

DIM. - 亡̀ ovvia conseguenza della def. 1 e della surgettività di $f$.

Segnaliamo, poichè dovremo adoperarlo, un ulteriore risultato.

Lema 7 - Supponiamo che (E, Z) sia uno spazio uniforme, che A sia una parte di $E$ e che $\eta_{A}$ sia la struttura uniforme indotta da $\Re_{\text {su }}$ A. Allora, se $\mathfrak{F}$ è un qualunque filtro su $A$, il filtro $\mathscr{U}_{A}$-inviluppato $\mathfrak{F}_{\mathscr{U}_{A}}$ generato da $\mathfrak{F}$

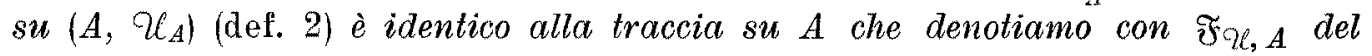
filtro $\mathfrak{Q}$-inviluppato $\mathfrak{F}_{\mathfrak{C}}$ generato da $\mathfrak{F} s u(E, \mathfrak{Z})$.

Drm. - Sia $X \in \mathfrak{F}_{\Upsilon C_{A} A}$ : esiste un'adiacenza $V$ di $(E, \mathcal{U})$ tale che, per un certo $F \in \mathfrak{F}$, sia $X=V(F) \cap A$. Detta $V_{A}$ la traccia di $V$ su $A \times A$ (cioè $\left.V_{A}=V \cap(A \times A)\right)$, risulta $X=V_{A}(F)$ e quindi, poichè $V_{A}$ è una adiacenza di $\left(A, \mathscr{\ell}_{A}\right)$, consegue $X \in \widetilde{\mho}_{\Upsilon_{A}}$. Dunque $\widetilde{\mho}_{\mathscr{C}_{A} A}$ è meno fine di $\mathfrak{F}_{\mathscr{K}_{A}}$.

Reciprocamente in maniera analoga si riconosce che $\mathfrak{F}_{\mathfrak{U}_{A}}$ è meno fine di $\mathfrak{F}_{\mathscr{L}, A}$ e da ciò consegue la tesi.

\section{§ 3. - Spazil prelindelöfiani.}

1. Si dovranno adoperare appresso alcune proprietà dei filtri che godono della proprietà descritta dalla seguente

DEF. 1. - Si dice che il filtro $\mathfrak{F}$ sull'insieme $E$ verifica la proprietà della interseaione numerabile se ogni intersezione numerabile di elementi di $\mathfrak{F}$ non è vuota. 
Equivalentemente, se ogni successione $\left(F_{n}\right)_{n \in N}$ di elementi di $\mathfrak{F}$ ha inter. sezione non vuota.

La seguente proposizione viene qui esplicitamente stabilita per comoditä del lettore, benchè, almeno in parte, debba considerarsi implicitamente nota.

Prop. 1. - Se ₹̀ è un filtro sull' insieme $E$ le seguenti proposizioni sono equivalenti:

a) $\mathfrak{F}$ verifica la proprietò dell' intersezione numerabile (def. 1),

b) per ogni ricovrimento numerabile $\left(A_{n}\right)_{n \in N}$ di $E$, esiste un $n \in N$ tale che la traccia di $\mathfrak{F}$ su $A_{n}$ sia un filtro verificante la proprietà dell' intersezione numerabile,

c) per ogni ricovrimento numerabile $\left(A_{n}\right)_{n \in N}$ di $E$, esiste un $n \in N$ tale che la traccia di $₹$ su $A_{n}$ sia un filtro.

Drm. - Supposta vera la $a),\left(A_{n}\right)_{n \in N}$ sia un ricovrimento di $E$. Per assurdo, se per ogni $n \in N$, esistesse una successione $\left(F_{n p}\right)_{p \in N}$ di elementi di $\mathfrak{F}$, tale che $A_{n} \cap\left(\underset{p \in N}{\cap} F_{n p}\right)=\varnothing$, sarebbe $A_{n} \subset \bigcup_{p \in N}\left(\complement F_{n p}\right)$ e quindi, insieme ad $\left(A_{n}\right)_{n \in N}$, la $\left(C F_{n p}\right)_{(n, p) \in N \times N}$ sarebbe un ricovrimento di $E$ e quindi sarebbe vuota l'intersezione della famiglia numerabile $\left(F_{n p}\right)_{(n, p) \in N \times N}$, contro la a). Dunque è vera la $b$ ).

Supposta vera la b) la c) ne è ovvia conseguenza.

Supponiamo vera la c) e, per assurdo, supponiamo ohe esista una successione $\left(F_{n}\right)_{n \in N}$ di elementi di $\mathfrak{F}$ ávente intersezione vuota. Consegue che $\left(\mathcal{G} F_{n}\right)_{n \in N}$ è un ricovrimento di $E$ e ciò è contro la $c$ ) poichè risulta $F_{n} \cap$ $n_{s}\left(C F_{n}\right)=\varnothing$ e $F_{n} \in \mathfrak{F}$ per ogni $n \in N$. Dunque è vera la $\left.a\right)$.

2. Si procede a formire alcune definizioni di uso particolarmente conveniente in quanto segue.

Innanzi tutto conveniamo ehe, salvo contrario esplicito avviso, si abbia che

CONVENZIONE 1. - E è uno spazio uniformizzabile.

Convenzione 2. - $\mathscr{A}(E)$ è la struttura uniforme di Tychonoff-C̄ech su $E$ secondo la terminologia di [2] § 3, def. 4. Pertanto $\mathscr{A}(E)$ è la più fine delle strutture uniformi di spazio precompatto su $E$ compatibili con la topologia di $E$. $\grave{E}$ noto (cfr. [2] § 3, def. 4) che $V$ è un'adiacenza dello spazio uniforme $(E, \mathcal{A}(E))$ se e solo se esiste un ricovrimento aperto finito $\chi(t$-riducibile (cfr. $[2$ § 3, def. 6) $\left(U_{k}\right)_{0 \leq k \leq n}$ tale che

$$
\bigcup_{k=0}^{n}\left(U_{k} \times U_{k}\right) \subset V
$$


Convenzione 3 - Detto, come al solito, $\boldsymbol{N}$ l'insieme degli interi naturali, lo zero incluso, indichiamo con w il numero cardinale di $\mathbf{N}$, ciò̀

$$
\omega=\operatorname{card}(\boldsymbol{N}) \text {. }
$$

(cioè $\omega$ è alef-zero).

Convenzione 4, - $\mathscr{L}_{\omega}(E)$ è la $\omega$-struttura uniforme su $E$ secondo la terminologia di $[3] \S 3$, def. 4. Pertanto, $\mathscr{A}_{\omega}(E)$. è la struttura uniforme su $E$ caratterizzata dal fatto che una parte $V$ di $E \times E$ è. un'adiacenza dello spazio uniforme $\left(E, \mathscr{A}_{\omega}(E)\right)$ se e solo se esiste un ricovrimento aperto, numebile, localmente finito ed $\ell-r i d u c i b l e ~\left(U_{n}\right)_{n \in N} d i E$ (cfr. [3] \$, def. 6) tale che

$$
\bigcup_{n \in N}\left(U_{n} \times U_{n}\right) \subset V
$$

Ovviamente $\mathscr{A}(E)$ è meno fine di $\mathscr{A}_{\omega}(E)$ e quindi, essendo la più fine delle strutture uniformi di spazio precompatto su $E$ compatibili con la topologia di $E, \mathscr{A}(E)$ risulta, esattamente, la struttura uniforme associala ad $\mathscr{A}_{\omega}(E)$ secondo la def. 1, \$ $2 d i[2]$.

OSSERvaZIONE. - Se a è un numero cardinale infinito e se $\mathscr{A}_{a}(E)$ è la $\mathfrak{a}$-struttura uniforme su $E, \mathscr{A}(E)$ seguita ad essere la struttura uniforme as. sociata ad $\mathscr{A}_{\mathfrak{a}}(E)$ in quanto $\mathscr{A}_{\omega}(E)$ è meno fine di $\mathscr{A}_{\mathfrak{a}}(E)$.

2. - Di particolare importanza per il seguito è la :

DEF. 2. - Chiamasi filtro completamente regolare su $E$ ogni filtro su $E$ che sia $\mathscr{A}(E)$-inviluppato (\$ 2, def. 1).

Osservazione 1. - Dunque il filtro $\mathfrak{F}$ su $E$ è completamente regolare se e solo se per ogni $F \in \mathfrak{F}$ esistono un $F^{\prime} \in \mathfrak{F}$, un insieme ohiuso $A$ un insieme aperto $B$ di $E$ tali che $F^{\prime} \subset A, A$ sia $U$-contenuto in $A$ e $B \subset F$ (cfr. [3] \& 3, def. 2 e [2] \& 3 , def. 4).

Osservazione 2. - Se a è un numero cardinale infinito, in forza della osservazione alla Convenzione 4 del n. 1, il filtro $\mathfrak{F}$ su $E$ è completamente regolare se e solo se esso a $\mathscr{A}(E)$-inviluppato ed in, particolare, se e solo se ̀̀ $\mathscr{A}_{\omega}(E)$-inviluppato.

Osservazione 3. - Se $A$ è un qualunque sottospazio di $E$, la traccia su $A$ di un qualunque filtro completamente regolare su $E$, se risulta un filtro, è completamente regolare (cfr. osservazione 1). 
DeF. 3. - Se $\mathfrak{B}$ è una base di filtro su E chiamasi filtro completamente regolare generato $d a \mathfrak{B}$, il filtro $\mathscr{A}(E)$-inviluppato generato dal filtro $s u \quad E d i$ cui $\mathfrak{B}$ è base secondo la def. 2 , §2.

Osservazione 1. - Richiamata la osservazione 1 della def. $1, F$ è un elemento del filtro completamente regolare generato da $\mathfrak{B}$ se $\theta$ solo se esistono un $F^{\prime} \in \mathfrak{B}$, un insieme chiuso $A$ ed un insieme aperto $B$ di $E$ tali che sia $F^{\prime} \subset A, A$ sia $U$-contenuto in $B$ e $B \subset F$.

Osservazione 2. - Se $\mathfrak{F}$ è un filtro completamente regolare su $E$ meno fine dell' ulteriore filtro $\mathfrak{F}^{\prime}$ su $E$, in forza del corollario alla prop. 4, §2, il filtro completamente regolare su $E$ generato da $\mathscr{F}^{\prime}$ è più fine di $\mathfrak{F}$.

Osservazione 3. - Se $\mathfrak{F}$ gode della proprietà dell'intersezione numerabile (def. 1), in forza della osservazione 2 alla def. 2 , §2 e della def. 3 qui sopra, anche il filtro completamente regolare da $\mathfrak{F}$, gode della stessa proprietà.

DEF. 4 - Chiamasi filtro completamente regolare massimale su E, ogni filtro $\mathscr{A}(E)$-inviluppato massimale su $E$ (cfr. def. $4 \$ 2$ e def. 2 di questo $\$$ ).

Osservazione 1. - Affinchè il filtro completamente regolare $\mathfrak{F}$ su $E$ sia massimale, per la prop. $11, \S 2$ e la def. 2 , è necessario e sufficiente che $\mathfrak{F}$ sia un filtro di Cauchy su $(E, \mathscr{A}(E))$.

n. 3. - In questo n. 3 si stabiliscono alcuni risultati dei quali si dorrà fare uso successivamente e nei quali interverranno le nozioni fino ad ora esposte.

Lemma 1. - Se ¿̀ è un sistema uniformizzante di Tukey sull'insieme $E$ (cfr. [3] \& 1, def. 9), se थ(ठ) è la struttura uniforme su E generata da $\delta[[3]$ $\S 1$, def. 8) e se $\mathfrak{F} \dot{e}$ un filtro su $E$, le seguenti proposizioni sono equivalenti:

a) $\mathfrak{~}$ e un filtro di Cauchy su $(E, \mathcal{U}(\mathcal{\delta}))$,

b) per ogni ricovrimento $\left(U_{t} h_{\in I} d i\right.$, esiste $\iota \in I$ tale che $U_{6} \in \mathfrak{F}$.

Dru. - a) implica b) Supponiamo vera la a) e sia $\left(U_{i} \in \mathbb{\text { un }}\right.$ elemento di $\delta$.

In forza della prop. $6, \S 1$ di $[3]$, esiste un'adiacenza $B$ di $(E, \mathscr{U}(\mathcal{C}))$ tale che $(B(x))_{x \in E}$ sia un raffinamento di $\left(U_{t}\right)_{: \in I}$. Per $a$ ) esiste an $F \in \mathfrak{F}$ tale che sia $F \times F \subset B$. Sia $x \in F$ e $\iota \in I$ tale che $B(x) \subset U_{\iota}$ : se ̀े $y \in F$, risulta

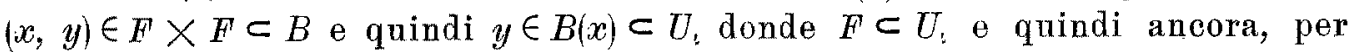
ua assioma di filtro, risulta $U_{t} \in \mathfrak{F}$. Cio implica $b$ ).

b) implica a). Supponiamo vera la $b$ ) e $V$ sia una qualunque adiacenza di $(E, \mathscr{U}(\circlearrowleft))$. Allora, in forza della def. 8 e del lemma $6, \& 1$ di [3] esiste un elemento $\left(U_{t}\right)_{i \in I}$ di $\delta$ tale che $\bigcup_{t \in I}\left(U_{t} \times U_{l}\right) \subset V$. Ciò, per $b$ ) implica $a$ ). 
LeMma 2. - L'insieme $\prod_{\omega}(E)$ dei ricovrimenti aperti numerabili ed ql-riducibili di $E$ (cfr. [3] \& 3, def. 6) è un sistema uniformizzante di TuKEY ([3] \& 1, def. 9) e la struttura uniforme $\chi\left(\eta_{\omega}(E)\right)$ da esso generata $([3] \& 1$, def. 8) è identica ad $\mathcal{A l}_{\mathrm{W}}(E)$.

Drm. - La tesi consegue osservando che $\bigcap_{\omega}(E)$ è esattamente l'insieme dei ricovrimenti aperti numerabili ed $\mathfrak{\Re}(E)$-riducibili di $E$ (ofr. [3] $\S 3$, deff. 5 e 6) dove $\mathfrak{R}(E)$ è il reticolo esteso universale su $E$ (ibidem, def. 3 ), e tenendo presente la prop. $5, \S 2$ di [3].

Osservazione. - Dunque, affinchè $V$ sia un'adiacenza di $\left(E, \mathscr{A}_{\omega}(E)\right)$ occorre e basta che esiste un ricovrimento aperto numerabile, $\ell$-riducibile (ma non necessariamente localmento finito) di $E$ tale che, indicatolo con $\left(U_{n}\right)_{n \in N}$, risulti :

$$
\bigcup_{n \in N}\left(U_{n} \times U_{n}\right) \subset V
$$

Prop. 2. - Se $\mathfrak{F} \grave{e}$ un filtro completamente regolare su $E$ (def. 2), le seguenti proposizioni sono equivalenti:

a) $\mathfrak{F}$ è un filtro completamente regolare massimale (def. 4) e verifica la proprietà dell'intersezione numerabile (def. 1),

b) $\Im$ è un elemento massimale dell' insieme dei filtri completamente regolari e verificanti la proprietò dell'intersezione numerabile,

c) Per ogni ricovrimento aperto numerabile ed $\chi$-riducibile $([3] \S 3$, def. 6) $\left(U_{n}\right)_{n \in N}$ di $E$, esiste un $n \in N$ tale che sia $U_{n} \in \mathfrak{F}$,

d) $\mathfrak{z}$ è un filtro di Cauchy sullo spazio uniforme $\left(E, \mathscr{A}_{\omega}(E)\right)$.

DiM: - a) inplica b). È ovvia.

b) implica c). Supponiamo vera la b) e sia $\left(U_{n}\right)_{n \in N}$ un ricovrimento aperto numerabile ed $\ell$-riducibile di $E$ e $\left(F_{n}\right)_{n \in N}$ sia una sua $\mathscr{\ell}$-riduzione. Dunque, secondo la def. $5, \S 3$ di $[3],\left(F_{n}\right)_{n \in N}$ è un ricovrimento chiuso di $E$ tale che, per ogni $n \in N, F_{n}$ sia $\eta$-contenuto in $U_{n}$. Poichè, per b); $\widetilde{\mho}$ verifica la proprietà dell'intersezione numerabile, per la prop. 1 equivalenza di a) e b), esiste un $n \in N$ tale che la traccia di $\widetilde{F}$ su $F_{n}$ sia un filtro verifi. cante la stessa proprietà sicchè posto $\mathscr{\mho}^{\prime}=\mathfrak{F} \cup\left\{F_{n}\right\}, \widetilde{F}^{\prime}$ risulta base di un filtro $\mathfrak{F}^{\prime \prime}$ su $E$ del pari verificante la proprietà dell' intersezione numerabile.

Ovviamente $\widetilde{\xi}$ è meno fine di $\mathfrak{F}^{\prime \prime}$ sicchè, in forza della osservazione 2 della def. 3 il filtro completamente regolare $\mathfrak{F}_{\omega}^{\prime \prime}$ generato da $\mathfrak{F}^{\prime \prime}$ è più fine di F e, per la osservazione 3 alla def. 3 , gode della proprietà dell' intersezione numerabile. In forza di $b$ ), tutto ciò, $\mathscr{F}$ essendo massimale, implica $\mathfrak{F}=\mathfrak{F}_{\omega}^{\prime \prime}$. Consegue che è $U_{n} \in \mathfrak{F}$. Infatti, $F_{n}$ è $\mathscr{U}$-contenuto in $U_{n}$ e ciò, per la osservazione 1 alla def. 3, dimostra l'asserto.

c) implica d). Consegue dai lemma 2 e 1. 
d) implica a). Sia vera la $d)$ e sia $\left(F_{n}\right)_{n \in N}$ una successione di elementi di $\mathfrak{F}$, che, ragionando per assurdo, supponiamo abbia intersezione vuola.

Poichè, per ipotesi, $\mathfrak{乛}$ è completamente regolare, in forza della def. 2 e della osservazione 1 ad essa, per ogni $n \in N$, esistono un $F_{n}^{\prime} \in \mathfrak{F}$, un insieme chiuso $A_{n}$ e un insieme aperto $B_{n}$ di $E$ tali che $F_{n}^{\prime} \subset A_{n}, A_{n}$ sia $\mathscr{U}$-contenuto in $B_{n}$ e $B_{n} \subset F_{n}$ : per un assioma di filtro, da $F_{n}^{\prime} \in \mathfrak{F}$ e $F_{n} \subset A_{n}$ consegue $A_{n} \in \mathfrak{F}$. Consegue $\bigcap_{n \in N} B_{n}=\varnothing$ e quindi, $\left(\mathbf{C} B_{n}\right)_{n \in N}$ 仓े un ricovrimento chiuso di $E$; inoltre per note proprietà della $\mathscr{\ell}$-inelusione risulta che $\mathbb{G} B_{n}$ ̀े $\mathscr{\ell}$-contenuto in $\mathbf{G} A_{n}$. Consegue ancora che $\left(\mathbf{C} A_{n}\right)_{n \in N} \grave{e}$ un ricovrimento aperto numerabile $\ell$-riducibile di $E$ e $\left(\mathbb{C} B_{n}\right)_{n \in N} \Theta$ una sua $\ell$-riduzione. In forza di d) e dei lemmi 2 e 1 , esiste $n \in N$ tale che $\mathrm{C} A_{n} \in \mathscr{F}$ il che è contro la $A_{n} \in \mathfrak{F}$ sopra osservata. Dunque la intersezione di $\left(F_{n}\right)_{n \in N}$ non può essere vuota e da ciò consegue la a).

Una conseguenza immediata della proposizione dimostrata è la seguente

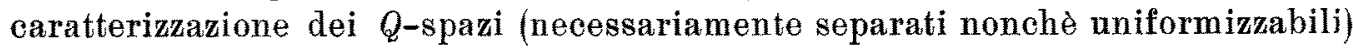
secondo HewITT:

Prop. 3 - Le seguenti proposizioni sono equivalenti:

a) $E \grave{e} \mathscr{A}_{\omega}$-completo,

b) ogni filtro completamente regolare massimale (def. 2) e verificante la proprietà dell' intersezione numerabile ha intersezione non vuota,

c) ogni elemento massimale dell'insieme dei filti completamente regolari e verificanti la proprietà dell'intersezione numerabile, ordinato per finezza, ha intersezione non vuota.

Osservazionw 1. - Dunque se $E$ è separato, in forza della osservazione alle prop. $5 \& 1$. la precedente a) può sostituirsi con: $E$ è un $Q$-spazio (cioè $E$ è realcompatto).

Osservazione 2. - In forza della prop. $10 \S 2$, nelle b) e c) di cui so. pra si può leggere anzichè «intersezione non vuota» l'altra «aderenza non vuota».

Osservazione 3. - Uno spazio topologico $E$ è di Lindelöf ( $\$ 1$ osservazione 1 al lemma 1) se e solo se ogni filtro avente base chiusa e verificante la proprieta dell' intersezione numerabile, ha intersezione non vuota. Dalla proposizione di cui sopra consegue ogni spazio di Lindelöf regolare è $A_{\omega}$-completo.

Ossenvazione 4. - La prop. 3 sopra stabilita può raffrontarsi con la seguente conseguenza delle deff. 2 e 4 e della prop. 18 del $\$ 2$.

Per $E$ le seguenti proprietà sono equivalenti:

a) $E$ è compatto, 
b) ogni filtro completamente regolare massimale su $E$ (def. 4) ha intersezione non vuota,

c) ogni filtro completamente regolare su $E$ (def. 2) ha intersezione non vuota. (Si tenga presente la prop. 10 del $\& 2$ ).

4. - Se $\mathfrak{F}$ è un filtro completamente regolare su $E$, in forza delle deff. 2 e 4 e della prop. $14 \S 2$, esiste un filtro completamente regolare massima. le su $E$ più fine di $\mathfrak{F}$.

Anche se si ammette che, in aggiunta, $\widetilde{f}$ verifichi la proprieta della intersezione numerabile, in generale non può affermarsi che, quale che sia $\mathfrak{F}$, esista un filtro completamente regolare massimale e verificante la proprietà dell' intersezione numerabile più fine di $\mathfrak{F}$, senza imporre una notevole condizione restrittiva sullo spazio uniformizzabile $E$.

In relazione a ciò ed in relazione strettissima con gli spazii chiamati «I-spaces» da R. W. BAGLex e J. D. Mo Kright JR., è la proprietà deseritta nella seguente def. 5. A tale proprietà si perverrà più spontaneamente dopo alcuni preleminari.

Lemma 3. - Supponiamo che Ẽ sia uno spazio uniformizzabile e che $E$ sia una sua parte ovunque densa e supponiamo che dette $\mathscr{A}_{\omega}(\tilde{E})$ e $\mathscr{A}_{\omega}(E)$ le rispettive $\omega$-strutture uniformi (cfr. n. 2 Convenzione 4 ) 'e detta $\mathscr{A}_{\omega}(\tilde{E})_{E}$ la struttura uniforme indotta da $\mathscr{A}_{\omega}(\tilde{E})$ su E risulti:

$$
\mathscr{A}_{\omega}(\tilde{E})_{E}=\mathscr{A}_{\omega}(E)
$$

Allora, supposto che $\left(F_{n}\right)_{n \in N}$ e $\left(G_{n}\right)_{n \in N}$ siano successioni di insieme chiusi e non vuoti e, rispettivamente, aperti $d i \tilde{E}$ tali che, per ogni $n \in N, F_{n}$ sia $\ell$-contenuto in $G_{n}([3] \S 3$, def. 2) relativamente ad $\tilde{E}$, risulta che:

$$
E \cap\left(\cap_{n \in N} G_{n}\right)=\varnothing \Rightarrow \bigcap_{n \in N} F_{n}=\varnothing
$$

Dim. - Lenotiamo con $\mathfrak{R}(\tilde{E})$ e $\mathfrak{R}(E)$ i reticoli estesi universali su $\tilde{E}$ e, rispett., su $E$ (cfr. [3] $\$ 3$, def. 3); risulta che

a) si ha $(F, G) \in \mathfrak{N}(\tilde{E})$ (rispett. $(F, G) \in \Re(E)$ se e solo se $F$ e $G$ sono insiemi chiusi il primo e aperto $i l$ secondo di $\tilde{E}$ (rispett. di $E$ ) ed $F \dot{e} \backslash$-contenuto in $G$ relativamente ad $\tilde{E}$ (rispett. ad E).

Ciò osservato, per ogni $n \in N$, poniamo:

$$
A_{n}^{\prime}=\mathfrak{G}_{\widetilde{E}} G_{n}, B_{n}^{\prime \prime}=\mathfrak{G}_{\widetilde{E}} F_{n} .
$$

Per $\alpha$ ), per là prop. $1 \S$ e def. 1 \& di [3], si ha che: 
$A_{n}^{\prime}$ ̀े $\ell$-contenuto in $B_{n}^{\prime \prime}$ relativamente ad $\tilde{E}$

ed inoltre esistono un insieme aperto $B_{n}^{\prime}$ ed un insieme chiuso $A_{n}^{\prime \prime}$ di $\tilde{E}$ tali che:

$$
\begin{gathered}
A_{n}^{\prime} \text { è } \mathscr{C} \text {-contenuto in } B_{n}^{\prime} \text { relativamente ad } \tilde{E}, \\
A_{n}^{\prime \prime} \text { ̀̀ } \mathscr{C} \text {-contenuto in } B_{n}^{\prime \prime} \text { relativamente ad } \tilde{E}, \\
B_{n}^{\prime} \subset A_{n}^{\prime \prime} .
\end{gathered}
$$

Ciò premesso sia $E \cap\left(\cap_{n \in N} G_{n}\right)=\varnothing$. Consegue $E=\bigcup_{n \in N}\left(A_{n}^{\prime} \cap E\right)$ ed inoltre, come è ovvio, $A_{n}^{\prime} \cap E$ è $\ell$-contenuto in $B_{n}^{\prime} \cap E$ relativamente ad $E$.

Dunque $\left(B_{n}^{\prime} \cap E\right)_{n \in N}$ è un ricovrimento aperto numerabile $\mathscr{\ell}$-riducibile del sottospazio $E$ e $\left(A_{n}^{\prime} \cap E\right)_{n \in N}$ è sua $\ell$-riduzione.

A causa del lemma $10, \S 2$ di [3], richiamata la $\alpha$ ) qui sopra, si riconosce che esiste un ricovrimento aperto numerabile, localmente finito ed $\ell$-riducibile del sottospazio $E$ che denotiamo con $\left(G_{n}^{*}\right)_{n \in N}$ tale che, per ogni $n \in \mathbb{N}$, $\operatorname{sia} G_{n}^{*} \subset B_{n} \cap E$. Orviamente per ogni $n \in N$ risulta

$$
G_{n}^{*} \subset A_{n}^{\prime \prime} \cap E .
$$

Supponiamo che $\left(F_{n}^{*}\right)_{n \in N}$ sia una $\mathscr{\ell - r i d u z i o n e ~ d i ~}\left(G_{n}^{*}\right)_{n \in N}$.

Per la prop. $1, \S 1$ di [4] risulta che $\left(F_{n}^{*}\right)_{n \in N} \dot{\theta} \mathcal{A}_{\omega}(E)$-uniformemente localmente finito nel sottospazio $E$ e quindi, per il lemma 2, § 1 ancora di [4] risulta che $\left(F_{n}^{*}\right)_{n \in N}$ e anche $S \mathcal{A}_{\omega}(\tilde{E})$-uniformemente localmente finito in $\tilde{E}$ (si tenga presente la (1)). Per ciò si ha

$$
\tilde{E}=\bar{E}^{(\sim)}=\bigcup_{n \in N}{\overline{F_{n}^{*}}}^{(\sim)}
$$

dove $-(\sim)$ denota aderenza relativa all'intero $\tilde{E}$.

Per ogni $n \in N$, richiamata la (4), si ha $F_{n}^{*} \subset G_{n}^{*} \subset A_{n}^{\prime \prime}$ e quindi, $A_{n}^{\prime \prime}$ es-

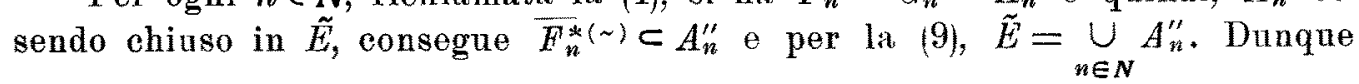
deve essere,

$$
\bigcap_{n \in N} F_{n}=Q_{\tilde{E}}\left(\bigcup_{n \in N} A_{n}^{\prime \prime}\right)=\varnothing
$$

e da cio la tesi,

LemMa 4. - Supposto come al solito che E sia uno spazio uniformizzabile le seguenti proposizioni sono equivalenti:

a) E è spazio di Lindelöf,

b) Ogni filtro completamente regolare (def. 2) e verificante la proprietà della intersezione numerabile (def. 1) su $E$ ha intersezione non vuota, 


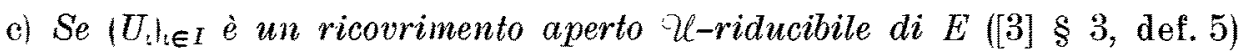
esiste una parle numerabile $H$ di $E$ tale che la sottofamiglia $\left(U_{i}\right)_{\in H}$ sia un ricovrimento di $E$.

Drm. a) implica b). È ovvia, poichè ogni filtro completamente regolare ha una base formata da insiemi chiusi

b) implica e). Supponiamo che $\left(U_{b}\right)_{\ell}$ sia un ricovrimento aperto $\ell-r \mathrm{i}$ ducibile di $E$ e $\left(F_{i}\right)_{\in I}$ sia una sua $\ell$-riduzione.

Ragionando per assurdo supponiamo ohe

a) se $H$ è una qualunque parte numerabile di $I$ risulti $E \neq \bigcup_{i \in H} U_{i}$.

Denotiamo con $\mathfrak{B}$ l'insieme delle parti $B$ di $E$ della forma

$$
B=\mathrm{O}\left(\bigcup_{i \in H} U_{t}\right) \text { con } H \text { parte numerabile di } I \text {. }
$$

Ovviamente, $\mathfrak{B}$ è base di un filtro $\mathfrak{F}$ su $E$, verificante la proprietà dell'intersezione numerabile. Se $\mathfrak{F}^{*}$ è il filtro completamente regolare su $E$ generato da $\mathfrak{F}$ (def. 3 ) anche $\mathfrak{F}^{*}$ verifica la proprietà dell'intersezione nume. rabile (def. 3, osservazione 3) A causa della b) risulta

$$
\bigcap_{F \in \Im^{*}} F \neq \varnothing
$$

Per ogni $t \in I$, essendo $F: q$-contenuto in $U_{t}$, si ha che $\complement U_{l}$ è $U$-contenuto in $C F_{\text {a }}$ e quindi per l'osservazione 1 alla def. 3 , si ha $G F_{\imath} \in \mathfrak{F}^{*}$ e ciò. poichè $\left(F, f_{i} \in I\right.$ è un ricovrimento di $E$, è contro la (1). Dunque $\alpha$ ) è falsa e c) $\grave{\theta}$ vera.

c) implica a). Supponiamo vera la c) e supponiamo che $\left(U_{\text {the }}\right.$ sia un ricovrimento aperto di $E$. Per ogni $x \in E$ esiste un $t_{x} \in I$ tale che $x \in U_{t_{x}}$ e poichè $E$ è uniformizzabile, in forza del lemma 6,83 di [3], esiste un insieme chiuso $F_{x}$ di $E$ tale che sia $x \in F_{x}$ ed $F_{x}$ sia $\ell$-contenuto in $U_{x_{x}}$. Dunque $\left(U_{b x}\right)^{x \in E}$ è un ricovrimento aperto $U$-riducibile di $E$ ed $\left(F_{x}\right)_{x \in E}$ è una sua $\mathscr{U}$-riduzione. Da c) consegue che esiste una parte numerabile $K$ di $E$ tale che $E=\bigcup_{x \in k} U_{x_{x}}$ : detto $H$ l'insieme degli elementi della famiglia $\left(t_{x}\right)_{x \in k}$, si ha $E=\bigcup_{t \in H}^{x \in k} U_{i}$. Da cio, $H$ essendo numerabile, la a).

6. - In ciò che segue adopereremo la

Convenzrone 5. - $\mathfrak{g}(E)$ denota l'insieme dei filtri completamente regolari (def. 2) e verificanti la proprietd dell' intersezione numerabile (def. 1).

DEF. - Chiamasi spazio prelindelöfiano ogni spazio uniformizzabile $E$ che verifichi l'assioma: 
(PL). - Per ogni $\mathfrak{F} \in \mathfrak{J}(E)$ esiste un $\mathfrak{F}^{*} \in \mathfrak{d}(E)$ massimale in $\mathfrak{d}(E)$ ordinato per «finezza».

La proprietà (PL) ha origine dalla proprietà consimile adoperata in [6]. La successiva prop. 11 giustificherà il termine "prelindelöfiano».

Prop. 4. - Le seguenti proprietà sono equivalenti per $E$ :

a) $E$ è prelindelöfiano (def. 5 ),

b) per:ogni $\mathfrak{F} \in \mathfrak{J}(E)$ esiste un filtro completamente regolare massimale $\mathfrak{F}^{*}$ che è in $\mathfrak{J}(E)$ ed è più fine $d i \mathfrak{F}$,

e) per ogni $\mathfrak{F} \in \mathfrak{I}(E)$ esiste un filtro completamente regolare $\mathfrak{F}^{*}$ su $E$ pì fine di $\mathfrak{F}$ e tale che per ogni ricovrimento aperto numerabile e U-riducibile $\left(U_{n}\right)_{n \in N} d i i_{\hat{S}} E$, esista $n \in N$ tale che $U_{n} \in \mathfrak{F}^{*}$,

d) per ogni $\mathfrak{F} \in \mathfrak{D}(E)$ esiste un filtro completamente regolare $\mathfrak{F}^{*}$ su $E$ piu fine di $\mathfrak{F}$ e che risulla di Cauchy per $\mathscr{A}_{\omega}(E)$,

$d i \mathfrak{F}$.

e) per ogni $\mathfrak{F} \in \mathfrak{J}(E)$ esiste un filltro di Cauchy su $\left(E, \mathscr{A}_{\omega}(E)\right)$ pì̀ fine

Drm. - L'equivalenza di a), b), c) e d) è conseguenza diretta della def. 5 e dalla prop. 2. Inoltre che d) implichi e) è banale e che e) implichi d) risulta da quanto segue.

Supponiamo vera la e) e sia $\mathfrak{F} \in \mathfrak{d}(E)$. In forza della osservazione 2 alla def. $2, \widetilde{F}$ è un filtro $\mathscr{A}_{\omega}(E)$-inviluppato. Per e) esiste un filtro di CAdoHY $\mathfrak{F}^{\prime}$ su $\left(E, \mathscr{A}_{\omega}(E)\right)$ più fine di $\mathfrak{F}:$ sia $\mathfrak{F}^{*}$ il filtro $\mathcal{A}_{\omega}(E)$-inviluppato generato da $\mathfrak{F}^{\prime}$. Poichè $\mathfrak{F}^{\prime}$ è più fine di $\mathfrak{F}$ ed $\mathfrak{F}$ è completamente regolare, per la osservazione 2 alla def. $3, \mathfrak{F}^{*}$ è più fine di $\mathfrak{F}$ e, per la prop. 5 del $\S 2$, $\mathfrak{F}^{*}$ è un filtro di CAUCHX su $\left(E, \mathscr{A}_{\omega}(E)\right)$ nella stesso tempo che, per la detta osservazione 2 alla def. 2, esso è completamente regolare.

Un prevedibile esempio di spazio prelindelöfiano è fornito dalla seguente proposizione:

Prop. 5. - Le seguenti proposizioni sono equivalenti:

a) $E$ è prelindelöfiano (def.5) ed $\mathscr{A}_{\omega}$-completo (ciò̀ $\left(E, \mathscr{A}_{\omega}(E)\right)$ è completo).

b) E è uno spazio di Lindelöf.

Drm. - a) implica b). Supponiamo vera la a) e sia $\mathfrak{F} \in \mathfrak{g}(E)$. Per la def. 5 , ed in forza della prop. 4 , esiste un filtro di CAUCHY $\mathfrak{F}^{*}$ su $\left(E, \mathcal{A}_{\omega}(E)\right)$ più fine di $\mathfrak{F}$. Poichè $E$ è $\mathscr{A}_{\omega}$ completo, $\mathfrak{F}^{*}$ converge e quindi $\mathfrak{F}$ ha almeno un punto aderente che, $\mathfrak{F}$ avendo una base formata da insiemi chiusi, appartiene all'intersezione di $\mathfrak{F}$. Ciò, per il lemma 4, dimostra b).

b) implica a). Supponiamo vera la b). Per l'osservazione 3 alla prop. 3 , $E$ ò $\mathscr{A}_{\omega}$-completo. Inoltre se $\widetilde{\jmath}$ è un filtro completamente regolare su $E$, per il lemma $2, \mathfrak{F}$ ha aderenza non vuota e quindi esiste un filtro $\mathfrak{F}^{*}$ su $E$ più 
fine di $\mathfrak{F}$ e convergente: dunque $\mathfrak{F}^{*}$ è un filtro di CAUCHY sn $\left(E, \not l_{\omega}(E)\right)$. Ciò, per la prop. 4 (a) equivale el) dimostra che ha a) di questa prop. 5 è vera.

Prop. 6 - Se E è C-numerabilmente compatto (ciò̀ pseudocompatto per [3] $\$ 8$ coroll. prop. 1) secondo la def. $1 . \$ 6$ di [3], allora È è prelindelöfiano.

Drm. - Per la osservazione alla convenzione $4, \mathscr{A}(E)$ è meno fine di $\mathscr{A}_{\omega}(E)$, mentre che per il lemma $2, \S 6$ di $[3]: \mathscr{A}_{\omega}(E)$ \& meno fine di $\mathscr{A}(E)$. Dunque è $\mathscr{A}_{\omega}(E)=\mathscr{A}(E)$. Per la def. 4 e per la prop. $14 \S 2$, esiste un filtro di $\mathrm{C}_{\mathrm{AUCHY}} \mathfrak{F}^{*}$ su $(E, \mathscr{A}(E))$ più fine di $\mathfrak{F}$. Dunque $\mathfrak{F}^{*}$ è un filtro di CatoHY su $\left(E, \mathscr{A}_{\omega}(E)\right)$ e quindi per la prop. 4 equivalenza di a) ed e), consegue la tesi.

Indichiamo ora alcune proprietà degli spazi prelindelöfioni.

Prop. 7 - Se $\left(E_{n}\right)_{n \in N} \grave{e}$ una successione di sottospazi prelindelofiani di $E$ della quale $E$ sia riunione, allora $E$ è prelindelöfiano.

DrM. - Denotiamo con $\mathfrak{F}$ un qualunque elemento di $\mathscr{J}(E)$. In forza della prop. 1, equivalenza di a) $\in$ b), esiste $n \in N$, tale che la traccia $\mathfrak{F}_{n}$ di $\mathfrak{F}$ su $E_{n}$ sia un filtro su $E_{n}$ verificante la proprietà della intersezione numerabile. Per l'osservazione 3 alla def. 2, $\widetilde{F}$ è un filtro completamente regolare sul sottospazio $E_{n}$ e, poichè il sottospazio $E_{n}$ è prelindelöfiano, esiste un filtro di CAUCHY $\mathfrak{F}^{\prime}$ su $\left(E_{n}, \mathscr{A}_{\omega}\left(E_{n}\right)\right.$ ) (dove $\mathscr{A}_{\omega}\left(E_{n}\right)$ è la $\omega$-struttara uniforme su $E_{n}$ (cfr. Convenzione 4) più fine di $\mathscr{F}$. Sia $\mathscr{A}_{\omega}(E)_{E_{n}}$ la struttura uniforme indotta da $\mathscr{A}_{\omega}(E)$ su $E_{n}: \mathscr{A}_{\omega}(E)_{E_{n}}$ è meno fine di $\mathscr{A}_{\omega}\left(E_{n}\right)$ e quindi $\mathfrak{F}^{\prime}$ è anche filtro di CAUCHY su $\left(E_{n}, \mathcal{A}_{\omega}(E)_{E_{n}}\right)$. Sia $\mathfrak{F}^{*}$ il filtro su $E$ che ha come base $\mathscr{F}^{\prime}$ (cioè generato da $\left.\mathfrak{F}^{\prime}\right)$ : evidentemente $\mathfrak{F}$ è meno fine di $\mathfrak{F}^{*}$ e $\mathfrak{F}^{*}$ è filtro di CAUCHY su $\left(E, \mathscr{A}_{\omega}(E)\right)$. Per la prop. 4 , equivalenza di a) ed e), consegue la tesi.

Prop. 8 - Se $f: E \rightarrow E^{\prime} \dot{e} u n '$ applicazione surgettiva e continua di $E$ sopra uno spazio uniformizzabile $E^{\prime}$, ogni volta che $E$ sia prelindelöfiano anche $E^{\prime}$ lo $\dot{e}$.

DrM. - Sia $\mathscr{A}_{\omega}\left(E^{\prime}\right)$ la $\omega$-struttura uniforme sı $E^{\prime}$ (Convenzione 4). Poichè $f$ è continua essa è un' applicazione uniformemente continua di $\left(E, \mathcal{A}_{\omega}(E)\right)$ su $\left(E^{\prime}, s \mathcal{A}_{\omega}\left(E^{\prime}\right)\right.$ in conseguenza del lemma $3, \S 3$ di [4]. Sia $\mathfrak{F}^{\prime}$ un filtro comple. tamente regolare verificante la proprietà dell' intersezione numerabile su $E^{\prime}$. Per la prop. $19 \S 2$ e per la def. $2, f$ essendo surgettiva, l'immagine reciproca $f^{-1}\left(\mathfrak{F}^{\prime}\right)$ di $\mathscr{F}^{\prime}$ per $f$ è base di un filtro $\mathfrak{F}$ su $E$ appartenente ad $\mathfrak{J}(E)$. Poichè $E$ è prelindelöfiano, per l'equivalenza di al ed e) nella prop. 4, esiste un filtro di $\mathrm{C}_{A U C H Y} \mathfrak{F}^{*}$ su $\left(E, \mathscr{A}_{\omega}(E)\right)$ più fine di $f^{-1}(\mathfrak{F})$ : la sua immagine $f\left(\mathfrak{F}^{*}\right)$ per $f$ è un filtro di CAUCHX su $\left(E^{\prime}, \mathscr{A}_{\omega}\left(E^{\prime}\right) \mid\right.$ più fine di $\mathcal{F}^{\prime}$ donde, ancora per la prop. 4 , a) equivale e), $E^{\prime}$ risulta prelindelöfiano.

Prop. 9 - Supponiamo che E sia uno spazio regolare (non necessariamente 
separato $=d i$ Hausdorff) normale (e quindi uniformizzabile) prelindelofiano e supponiamo che $F$ sia un insieme chiuso di E. Allora il sottospazio $F$ è prelindelöfiano.

Dim. - Siano $\mathscr{A}_{\omega}(E)$ le $\omega$-strutture uniformi su $E$ e, rispettivamente, su $F$, secondo la Convenzione 4. Detta $\mathscr{A}_{\omega}(E)_{F}$ la struttura uniforme indotta da $\mathscr{A}_{\omega}(E)$ su $F$, poichè $E$ è normale, in forza della prop. $7, \S 3$ di [3], risulta

$$
\mathscr{A}_{\omega}(E)_{F}=\mathscr{A}_{\omega}(F) \text {. }
$$

Sia $\mathfrak{f} \in \mathfrak{J}(F)$ ('analogo di $\mathscr{F}(E)$ della Convenzione 5) e sia $\mathscr{F}^{\prime}$ il filtro su $E$ che ha come base $\mathfrak{F}$ e sia $\mathfrak{F}_{\omega}^{\prime}$ il filtro complementare regolare generato da $\mathfrak{F}^{\prime}$ nell'intero spazio $E$ (def. 3). Al pari di $\mathfrak{F}$, prima $\mathfrak{F}^{\prime}$ e poi $\mathfrak{F}_{\omega}^{\prime}$ verificano la proprietà dell'interesse numerabile. Per il lemma $7 \& 2$, la traceia $\mathfrak{F}_{\omega F}^{\prime}$ di $\mathfrak{F}_{\omega}^{\prime}$ su $F$ è identica ad $\mathfrak{F}$.

Poichè $E$ è prelindelôfiano, per l'equivalenza di a) e d) nella prop. 4, esiste un filtro completamente regolare di CAUCHY $\mathfrak{F}^{*}$ su $\left(E, \mathscr{A}_{\omega}(E)\right)$ più fine di $\mathfrak{F}^{\prime}$. Riconosciamo che la traccia $\mathfrak{F}_{F}^{*}$ di $\mathfrak{F}^{*}$ su $F$ è nn filtro. Ragionando per assurdo, supponiamo che esista un $H \in \mathfrak{F}^{*}$ tale che $F \cap H=\varnothing$ : poichè $\mathfrak{F}^{*}$ ha una base chiusa, si può supporre che $H$ sia chiuso. Allora $G H$ è aperto e contiene $F$ e, poichè $E$ è normale, $F$ risulta $\ell$-contenuto in $G H$ (ofr. [3] \& 3, prop. 1, osservazione) e quindi per l'osservazione 1 alla def. 3, risulta $\mathbb{C} H \in \mathfrak{F}_{\omega}^{\prime}$ ed $\mathfrak{F}_{\omega}^{\prime}$ essendo meno fine di $\mathfrak{F}^{\prime}$, si ha $\mathrm{C} H \in \mathfrak{F}^{*}$ contro la $H \in \mathfrak{F}^{*}$. Dunque $\mathfrak{F}_{F}^{*} \grave{e ̀ ~ u n ~ f i l t r o ~ s u ~} F$ che è un filtro di CAJCHY per $s \mathcal{A}_{\omega}(E)_{F}$ ossia, per (1), filtro di CAUchY su $\left(F, \mathscr{A}_{\omega}(F)\right)$, eiò che, $\mathfrak{F}_{F}^{*}$ essendo più fine di $\mathfrak{F}_{\omega F}^{\prime}=\mathfrak{F}$, dimostra la tesi in forza della prop. 4, a) implica e).

Sussiste ora la proposizione oonclusiva che procediamo a dimostrare con ogni dettaglio.

Prop. 10 - Supponiamo che $\tilde{E}$ sia uno spazio uniformizzabile e suppo* niamo che $E$ sia una parte ovunque densa di $\tilde{E}$ tale che, dette $\mathcal{A}_{\omega^{\prime}}(\tilde{E})$ e $\mathcal{A}_{\omega}(E)$ le $\omega$-strutture uniformi su $\tilde{E}$ e, rispett., su $E$ (Convenzione 4 ) la $\mathscr{A}_{\omega}(\tilde{E})$ sia una struttura uniforme di spazio completo e, detta $\mathscr{A}_{\omega}(\tilde{E})_{E}$ la struttura uniforme indotta da $\mathscr{A}_{\omega}(\tilde{E})$ su $E$, risulti:

$$
\mathscr{R}_{\omega}(\tilde{E})_{E}=\mathscr{A}_{\omega}(E)
$$

Allora le seguenti sono equivalenti:

a) ogni $\mathfrak{F} \in \mathfrak{I}(E) \grave{e ̀ ~ m e n o ~ f i n e ~ d i ~ a l i m e n o ~ u n ~} \mathfrak{F} \in \mathfrak{J}(E)$ massimale su $\mathfrak{J}(E)$ ordinato per «finezza».

b) $\tilde{E}$ è uno spazio di Lindelöf.

DiM. - a) implica b). Sia vera la a) e denotiamo con $\mathfrak{F}$ un qualanque 
elemento di $\mathfrak{g}(\tilde{E})$ (analogo di $\mathfrak{d}(E)$ della Convenzione 5); a causa della equivalenza di a) e b) nel lemma 4 , la presente b) sarà dimostrata stabilendo che $\mathfrak{F}$ ha intersezione non vuota.

Poichè $\mathfrak{F}$ ha una base formata da insiemi aperti ed $E$ è ovunque denso in $\tilde{E}$, la traccia $\mathfrak{F}_{E}$ di $\widetilde{F}$ su $E$ è un filtro completamente regolare. Poichè $\mathfrak{F}$ ha anche una base formata da insiemi chiusi, ricordata l'osservazione 1 alla def. 2, in forza del lemma $3, \mathscr{F}_{E}$ verifica la proprietà dell' intersezione numerabile al pari di $\mathfrak{F}$. Dunque è $\mathfrak{F}_{E} \in \mathfrak{J}(E)$ e quindi per a) esiste un $\mathfrak{F}^{*} \in \mathfrak{S}(E)$ massimale nel modo descritto da a). In forza della equivalenza di b) e d) nella prop. $4, \mathfrak{F}^{*}$ è un filtro di CAUCHY su $\left(E, \mathscr{A}_{\omega}(E)\right)$ e quindi, per l'ipotesi (1), un filtro di CaUCHY su $\left(E, \mathscr{A}_{\mathcal{C}}(\tilde{E})_{E}\right)$. Pertanto $\mathfrak{F}^{*}$ è base di un filtro di CADOHY su $\left(\tilde{E}, \mathscr{A}_{\omega}(\tilde{E})\right)$ che denotiamo con $\tilde{\mathfrak{F}}^{*}$ e sia $\tilde{\mathfrak{F}}_{\omega}^{*}$ il filtro completamente regolare su $\left(\tilde{E}, \mathscr{A}_{\omega}(\tilde{E})\right)$ generato da $\tilde{F}^{*}$. Per la prop. 5 , $\$ 2$, tale $\tilde{\mathfrak{F}}_{\omega}^{*}$ è un filtro di Caudhy su $\left(\tilde{E}, \mathscr{A}_{\omega}(\tilde{E})\right)$. Poichè, per ipotesi, $\left(\tilde{E}, \mathscr{A}_{\omega}(\tilde{E})\right)$ è completo, risulta:

$$
\cap F \neq \varnothing \text { per } F \text { variabile in } \tilde{\mathfrak{F}}_{\omega}^{*}
$$

A sua volta $\widetilde{F}_{E}$ è base di un filtro su $\tilde{E}$ che denotiamo con $\tilde{\mathfrak{F}}_{E}$.

Poichè è $\mathfrak{F} \subset \tilde{\mathfrak{F}}_{E} \subset \tilde{F}^{*}$, in forza dell'osservazione 2 alla def. 3 , si ha $\mathfrak{F} \subset \tilde{\mathfrak{F}}_{\omega}^{*}$ e quindi, per la (2), l'intersezione di $\mathfrak{F}$ non è vuota. Dunque è vera la b).

b) implica a). Supponiamo vera la b) e supponiamo che $\mathfrak{F}$ sia un filtro in $\mathfrak{J}(E)$ (Convenzione 5): per la osservazione 2 alla def. 2 , $\mathscr{F} \grave{e ̀ ~} \mathscr{A}_{\omega}(E)$-inviluppato.

Sia $\tilde{\mathscr{F}}$ il filtro su $\tilde{E}$ generato da $\widetilde{F}$ e sia $\tilde{F}_{\omega}$ il filtro $\mathcal{A}_{\omega}(\tilde{E})$-inviluppato su $\tilde{E}$ generato da $\tilde{\widetilde{F}}$ : per il lemma $7, \& 2$, la traccia $\tilde{\mathfrak{F}}_{\omega E}$ di $\tilde{\mathfrak{F}}_{\omega}$ su $E$ è identica al filtro $\mathscr{A}_{\omega}(E)$-inviluppato su $E$ generato da $\mathfrak{F}$ cioè, per la prop. $4 \S 2$, il filtro $\mathfrak{F}$ stesso (che per ipotesi è $\mathscr{A}_{\omega}(E)$ inviluppato; osservazione 2 alla def. 2).

Osserviamo che $\tilde{\mathfrak{F}}$, al pari di $\widetilde{F}$ verifica la proprietà dell'intersezione numerabile e quindi anche $\tilde{\mathfrak{F}}_{\omega}$ la verifica. Dalla equivalenza di a) e bj nel lemma 4 esiste un punto $x$ di $\tilde{E}$ appartenente all'intersezione o, se si vuole, all'aderenza di $\tilde{\mathfrak{F}}_{\omega}$. Consegue che esiste un filtro $\widetilde{\mathfrak{F}}$ su $\tilde{E}$ convergente verso $x$ e più fine di $\tilde{\widetilde{F}}_{\omega}$. Dunque detto $\mathfrak{B}(x)$ il fỉltro degli intorni di $x$ in $E$ risulta

$$
\mathfrak{B}(x) \text { meno fine di } \overline{\mathfrak{F}}
$$

$$
\tilde{\mathfrak{F}} \text { meno fine di } \tilde{\mathfrak{F}} \text {. }
$$

Sia $\widetilde{F}_{\omega}$ il filtro $\mathscr{A}_{\omega}(\tilde{E})$-inviluppato su $\tilde{E}$ generato da $\tilde{\mathfrak{F}}_{\omega}$. Da $(3)$ e $(4)$ e 
per la osservazione alla prop. $12 \S 2$, poichè $\mathfrak{S}(x)$ e $\tilde{\mathfrak{F}}_{\omega}$ sono $\mathscr{A}_{\omega}(E)-$ inviluppati, per la osservazione 2 alla def. 3, risulta: $\mathfrak{B}(x)$ meno fine di $\widetilde{F}_{\omega}$ $\tilde{\mathfrak{F}}_{\omega}$ meno fine di $\widetilde{\mathfrak{F}}_{\omega}$.

La traccia $\widetilde{\mho}_{\omega E}$ di $\widetilde{\widetilde{F}}_{\omega}$ su $E$ è un filtro poichè $E$ è ovunque denso in $\tilde{E}$ $\tilde{\widetilde{F}}_{\omega}$ ha una base formata da insiemi aperti.

Dalla (5) consegue che $\widetilde{\mathfrak{F}}_{\omega}$, oltre ad essere completamente regolare su $\tilde{E}$ converge verso $x$ e quindi è un filtro di CaUchy su $\left(\tilde{E}, \mathscr{A}_{\omega}(\tilde{E})\right)$. Pertanto, $\widetilde{\widetilde{F}}_{\omega E}$, oltre ad essere completamente regolare sul sottospazio $E$, è un filtro di CAUCHY su $\left(E, \mathscr{A}_{\omega}(E)\right)$ in forza di $(1)$.

A causa della equivalenza di b) e d) nella prop. 4, si ha $\widetilde{\mathfrak{F}}_{\omega E} \in \mathfrak{J}(E)$ ed è massimale (cfr. la a)). Inoltre, richiamata la (6), risulta

$$
\tilde{F}=\widetilde{\mathfrak{F}}_{\omega E} \text { meno fine di } \widetilde{\mathfrak{F}}_{\omega E} \text {. }
$$

Dunque la a) è vera.

Ed ora, dimostrata la prop. 10, non e'è che da far ricorso alla definizione dell' $\mathcal{A}_{\omega}$-completamento di uno spazio nniformizzabile $E$, per concludere:

Prop. 11. - Se lo spazio E è uniformizzabile separato, le seguenti proposizioni sono equivalenti:

a) $E$ è prelindelöfiano (def. 5),

b) $\nu_{\omega}(E)$ è uno spazio di Lindelöf.

In particolare, se $E$ è separato, $U_{\omega}(E)$ è l'estensione di Hewirc di $E$ e quindi $E$ è prelindelöfiano se e solo se l'estensione di Hewıtr di $E$ è uno spazio di LTNDELöF.

Si desidera segnalare che, in conseguenza di quest' ultima osservazione, o, se si vuole della prop. 11, per $E$ separato si può dare una agevole dimostrazione indiretta delle precedenti prop. $7,8,9$.

\section{BIBLIOGRAFIA}

[1] Alnsen E. M. a Fenstad J. E., A note on completion and compactification; "Math. Seand.», vol. $8(1960)$ pp. $97-104$.

[2] Aquaro G, Strutture uniformi di spazio precompatto, «Ann. Scuola Norm. Sup. di Pisa», serie III, vol. XI (1957) pp. 149-181.

[3] - -, Ricovrimenti aperti e strutture uniformi etc. .. : «Ann. di Mat. pura ed applic.» (IV), vol. XLVII (1959) pp. 319.390. 
[4] - -, Completamenti di spazii uniformi, "Ann di Mat. pura ed applic," (IV), vol. LVI (1961) pp. 87-98.

[5] - -, Spazii collettivamente normali ed estensione etc..., «Riv. Mat. Univ. Parna» (2) rol. 2 (1961) pp. 77.90.

[6] BAgLeY R. W. e MCKNIGHT J. D. JR., On Q-spaces and collections of closed sets ete..., «Quart. J. Math. Oxford. Ser. (2) vol. 10 (1959) pp. 238.235.

[7] Bourbaki N., Algèbre, Actual. Scient. et Ind. n. 934-1144 (1951) e n. 1032 (1947), Hermann e Co., Paris.

[8] - - Topologie Générale, Actual Scient. et Ind. n. 858.1142 (195̃1) n. 1045 (nouv. edit.) (1958), Hermann e Co., Paris.

[9] Grlmiman L. e J ERIson M, Rings of continuous functions, Van Nostrand Princeton (N. J.) (1960).

[10] KowaLski H.-J., Topologisehe Räume, Birkauser Ver. Basel-Stutgart (1961).

[11] SAMuer P., Ultrafilters and compactification of uniform spaces, «Trans. Amer. Math. Soc.» rol. 64 (1948) pp. $100 \cdot 132$. 